\title{
Cell Association in Dense Heterogeneous Cellular Networks
}

\author{
Mohammad G. Khoshkholgh, Keivan Navaie, Senior Memeber, IEEE, \\ Kang G. Shin, Life Fellow, IEEE, Victor C. M. Leung, Fellow, IEEE
}

\begin{abstract}
Coverage evaluation of heterogeneous multi-tier cellular networks (HetNets) is often based on simplifying assumptions on cell association (CA): the resource required by, and practical limitations of pilot measurements are overlooked. Also, the base station (BS) providing the strongest signal-to-interference ratio among all BSs is always the serving BS (an ideal CA (iCA)). Consequently, the resultant analysis falls short of characterizing HetNets' coverage in practical settings. We therefore propose an analytical framework for modeling a practical CA ( $\mathrm{pCA}$ ) by considering pilot measurement, pilot sensitivity at the users, and the number of pilot measurements, $K_{P}$. Using tools from stochastic geometry, we obtain the coverage with pCA in both Rayleigh and Nakagami environments. We propose an algorithm to obtain the optimal $K_{P}$ and its partitioning among the BSs in different tiers that maximizes the coverage. Our analysis provides key insights in designing dense HetNets. For dense networks, scale invariance achievable under iCA is shown unsustained with pCA. Also, dense HetNets are pilot-neutral, and hence their performance is not affected by pilot sensitivity. Our extensive simulations confirm the accuracy of our analysis and the proposed algorithm, and demonstrate the effect of pCA in comparison with iCA.
\end{abstract}

Index Terms—Cell association, coverage probability, densification, HetNets, pilot measurement, Poison Point Processes, stochastic geometry.

\section{INTRODUCTION}

$\mathrm{D}$ ENSIFICATION is increasingly used to meet the rapidly growing demand of wireless data communications in cellular networks [1,2]. Densification in cellular networks results in complex architectures of heterogenous networks (HetNets) in which, without careful cell association (CA), the expected performance improvements are often unattainable [3-6].

Conventional solutions often advocate association with the BS having the strongest signal, or equivalently with the nearest BS, assuming fading averages out. Such an association may cause coverage deterioration as well as load imbalance across BSs $[4,7]$ because densification often increases the received signal power at the user equipment (UE), but it may also impose severe intercell interference (ICI) that may eventually cancel out the improved signal strength.

To address this issue, researchers proposed techniques that consider use of the signal-to-interference ratio (SIR) or functions thereof - instead of the received signal strength - for CA. In such techniques, a UE is associated with the BS that provides the highest received SIR. Such CA techniques are often referred to as the max-SIR CA

- M. G. Khoshkholgh, and V. C. M. Leung (m.g.khoshkholgh@gmail.com, vleung@ece.ubc.ca) are with the Department of Electrical and Computer Engineering, the University of British Columbia, Vancouver, BC, Canada V6T 1Z4; K. Navaie (k.navaie@lancaster.ac.uk) is with the School of Computing and Communications, Lancaster University, Lancaster, United Kingdom LA1 4WA; K. G. Shin (kgshin@umich.edu) is with the Department of Electrical Engineering and Computer Science, University of Michigan, Ann Arbor, MI 48109-2121, U.S.A. Part of this paper was presented in the IEEE VTC'16-fall, Sep. 2016, Montreal, Canada. rule [7].

In HetNets with a large number of BSs and UEs, the effect of the CA rule on network performance is of practical importance, and has been an active research topic in recent years, see, e.g., [7-10]. Such efforts often adopt tools from stochastic geometry and Poisson Point Processes (PPP) $[11,12]$ to model the network. Compared to the conventional hexagonal grid and Wyner models, such techniques result in a tractable analysis which sheds light on the engineering and design aspects of networks which are otherwise unknown. Randomness and heterogeneity of HetNets are also shown to be effectively captured by PPP models, see, e.g., [13-17].

Large-scale measurements and industry-scale simulations in $[13,18]$ also confirm that the PPP models result in accurate estimation of the SIR distribution in cellular networks. It is further shown in $[15,16]$ that a simple 1-3 (dB) SIR shift is enough to match the SIR distribution obtained with PPP models with the one obtained from a grid model. The analysis and simulation results presented in [19] also show that any grid setting can be adequately modeled as a PPP due to the wireless channel shadowing effect. Based on the above observations, we adopt PPP to model HetNets.

For single-antenna (SISO) $K$-tier HetNets with maxSIR CA, the authors of [7] derived, for the first time, closed-form expressions for the coverage probability, achievable rate, and average load. The framework in [7] was later extended in [8] to the coverage probability of multi-antenna (MIMO) downlink with spacedivision multiple access (SDMA). Note that compared to the SISO systems, obtaining post-processing SIR in 
MIMO systems involves fading distributions other than Rayleigh, for instance, Nakagami. This results in very complex, often intractable, SIR distributions. Instead, approximations are made for the coverage probability in [8] by borrowing techniques from stochastic ordering. Later, the authors of [10] used the same approach as in [7] to explore the spectral efficiency of two-hop communications in cellular networks. The results in [7] were also used in [9] to improve the cost-efficiency of HetNets.

The coverage performance of a HetNets with various CA rules was investigated in [20]. Considering a general fading model, max-SIR CA, the closest BS CA (cCA) (whereby the closest BS is associated with the UE) and several variations of the maximum instantaneous received power were investigated in [20], where coverage probability was obtained as a set of integral expressions. Furthermore, the authors of $[21,22]$ investigate the SINR in HetNets and evaluate the $k$-coverage probability, i.e., the probability that $k$ BSs can potentially support the typical UE.

The analysis in all of the above mentioned investigations is based on a very limiting assumption on the CA rule: the best $\mathrm{BS}$ is selected out of the entire pool of BSs in the network. We call such a CA rule the ideal CA (iCA). This implies that in reality, however, to find the best $\mathrm{BS}$, a UE requires to measure the received pilot signals corresponding to all BSs, which may not be practically attainable.

Here we analyze a practical CA model and assume that a UE measures the received pilot signal corresponding to a limited number of neighboring BSs, $K_{P} \geq 1$. Association with near BSs is preferred to those located far away from the UE as they have heavy path-loss attenuation and may incur a high rate of handovers. The received pilot power is affected by the path-loss and also depends on the BSs' transmission power, which might vary significantly across different tiers of BSs. Due to UEs' PHY-layer configurations, only pilots that are strong enough - larger than a given threshold, $\gamma>0$, that we call pilot sensitivity - are considered detectable. Ignoring the received pilot power as in $\mathrm{iCA}$, it is often impossible to specify occasions on which a nearby BS (pico/femto BSs) with a low transmit power could be associated with a UE.

The main objective of this paper is to investigate the impact of pilot measurements on the coverage performance. In practice, CA is based on periodic measurements of pilot signal quality in a time-slotted setting, and comparing it with a pilot sensitivity threshold. Therefore, in addition to the path-loss attenuation, main limiting factors for the number of pilots that could be measured are pilot sensitivity as well as the time required for pilot measurement. Each received pilot signal is measured over a time interval, thus limiting the available time for data transmission.

There are also other factors that affect the CA performance, including (i) Transmit power of the BSs which is often limited, and thus the power allocated to the pilot signal power is also required to be carefully balanced against the remaining power to be allocated to data transmission, and (ii) Pilot contamination, which is caused by a pilot signal re-use policy, where the same pilot signal is re-used by multiple BSs in the coverage area. Here, we ignore these factors for brevity.

The impact of CA on the coverage performance of HetNets is also investigated in [23]. The CA considered in [23] is specifically designed for load balancing, where the closest macro-BS is chosen where there is no femtoBS in the vicinity of the UE that provides the highest SIR. Note that [23] only considers Rayleigh fading and ignores the effect of pilot measurements. The impact of CA on the network performance was also investigated in [24], where a heuristic association technique was developed. The technique in [24] selects the associated tier for each UE based on "max-ratio association policy". Under this policy, for each UE a tier is selected in which the UE's distance to its nearest BS is smaller than that in other tiers, while its distance to the second-nearest BS in that tier is larger than that in other tiers. The closest BS of the selected tier is then considered as the serving BS. The work in [24] assumes that each tier has its own exclusive bandwidth. Furthermore, the work of [24] did not consider pilot sensitivity of UEs and resource consumption of pilot measurements. Both [23], and [24] did not consider optimal selection of $K_{P}$ and its partitioning, either.

In this paper we propose a general model for CA, namely practical $C A$ (pCA) as opposed to the ideal $C A$ (iCA) model in [7]. The main parameters in this model in addition to $\gamma$ are the size of the candidate set from which each UE selected its serving BS, and the partitioning of the candidate set for different tiers of BSs. This makes the following main contributions. We (i) propose an analytical framework for modeling pCA; (ii) this model is then used to obtain the coverage performance of HetNets. We then (iii) formulate the pCA design as an optimization problem to maximize the coverage probability of the HetNet, and propose a greedy algorithm to obtain these parameters including the size of the candidate set and its optimal partitioning; and (iv) adopting techniques from stochastic geometry and PPP, we obtain closedform expressions and accurate approximations for the coverage probability of a HetNet for a generic pCA in both Rayleigh and Nakagami fading environments; and further show that $(v)$ dense HetNets are pilot-neutral, i.e., their coverage performance is not a function of pilot sensitivity, $\gamma$, and (vi) scale-invariance in HetNets with iCA in which the spectral efficiency grows with densification and the coverage probability stays stable, is not sustained in dense HetNets.

Our simulation results show that compared to iCA, as a theoretical (thus impractical) benchmark, pCA always results in coverage performance loss. We also show that (i) for both Rayleigh and Nakagami fading environments, there exist an optimal size of the candidate set, $K_{P}$, that minimizes the coverage loss of the HetNet with 
pCA compared to iCA; (ii) unbalanced partitioning of $K_{P}$ among the tiers deteriorates the coverage probability, especially in dense HetNets; (iii) in dense HetNets (or equivalently where $\gamma$ is large enough), the coverage performance of the $\mathrm{pCA}$ is significantly higher that that of cCA; (iv) in Nakagami fading environments, the coverage probability is smaller than that of Rayleigh fading, particularly in sparse networks; and $(v)$ optimized coverage performance in a Nakagami fading environment is archived with a lower $K_{P}$ than in a Rayleigh fading environment.

The rest of this paper is organized as follows. Section 2 presents our system model as well as the mathematical model for pCA. Then, in Section 3 we devise an optimization problem and a greedy algorithm to obtain the optimal pCA parameters. In Section 4, we present a coverage performance analysis for HetNets with pCA in both Rayleigh and Nakagami fading environments. The impacts of densification on the coverage performance are investigated in Section 5 followed by the simulation results in Section 6. The paper concludes with Section 7.

\section{System MOdel}

Consider downlink communication in a $K$-tier HetNet. As in $[7,25]$, we model the network by $K$ tiers (classes/technologies) of randomly located BSs in the coverage area. Each tier $i$ is specified by 3 tuples $\left(\lambda_{i}, P_{i}, R_{i}\right)$ where $\lambda_{i}$ is the $\mathrm{BSs}^{\prime}$ spatial density (per unit area), $P_{i}$ is per-frame average transmission power in Watts, and $R_{i}$ is the $i$-th tier maximum data rate (nat/sec/Hz). BSs in tier $i$ are spatially distributed according to a homogenous Poisson Point Process (PPP), $\Phi_{i} \in \mathbb{R}^{2}$, with spatial density of $\lambda_{i} \geq 0$, where the processes are mutually independent. UEs are distributed according to a homogenous PPP $\Phi_{U}$, independent of sets $\Phi_{i}, \forall i$. We also set $\Phi=\bigcup_{i} \Phi_{i}$.

We consider slotted time and narrow-band block fading which remains constant during a time slot. The main performance metric is the coverage probability which is measured from the perspective of a UE located at the origin, which we call a typical UE. Due to the stationarity of the point processes, Slivnyak's Theorem [11] guarantees the performance at the origin measured by the typical UE to represent the spatial performance of the network.

Our focus in this paper is on dense HetNets with universal frequency reuse which are interference-limited. Thus, we ignore the thermal noise effect. The signalto-interference ratio (SIR) experienced at the typical UE served by BS $x_{i}$ is

$$
\operatorname{SIR}_{x_{i}}=\frac{P_{i}\left\|x_{i}\right\|^{-\alpha} h_{x_{i}}}{\sum_{j=1}^{K} I_{j}}
$$

where $\alpha>2$ is the path-loss exponent, and $\left\|x_{i}\right\|$ represents the Euclidean distance. We further set $\check{\alpha}=\frac{2}{\alpha}$. Let $\left\{h_{x_{j}}\right\}$ denote channel power gains which are independent and identically distributed random variables. The
TABLE 1

List of Parameters and Notations

\begin{tabular}{|c|c|}
\hline Symbol & Description \\
\hline$K$ & Number of tiers \\
\hline$\Phi_{i}=\left\{x_{i}\right\}$ & $\begin{array}{l}\text { BSs of tier } i \text { with density } \lambda_{i} \text {, transmission power } P_{i} \text {, } \\
\text { data rate threshold } R_{i}\end{array}$ \\
\hline$\gamma$ & $\begin{array}{l}\text { Required pilot strength for detection at UEs (pilot } \\
\text { sensitivity) }\end{array}$ \\
\hline$\alpha \in(2,6]$ & Path-loss exponent $\left(\check{\alpha} \triangleq \frac{2}{\alpha}\right)$ \\
\hline$C(\alpha)$ & $\triangleq \check{\alpha} \pi^{2} \csc (\check{\alpha} \pi)$ \\
\hline$\kappa_{j}($ resp. $\kappa)$ & $\triangleq \lambda_{j} P_{j}^{\check{\alpha}}$ (resp. $\triangleq \frac{C(\alpha)}{\pi} \sum_{j=1}^{K} \kappa_{j}$ ) \\
\hline$\hat{\kappa}_{i}$ & $\triangleq \frac{\lambda_{i}}{\kappa}\left(\frac{P_{i}}{\hat{\beta}_{i}}\right)^{\check{\alpha}}$ \\
\hline$h_{x_{i}}$ & fading power gain between BS $x_{i}$ and the typical UE \\
\hline$M_{i}$ & Nakagami parameter of tier $i$ \\
\hline$I_{j}=\sum_{x_{j} \in \Phi_{j}} P_{j}\left\|x_{j}\right\|^{-\alpha} h_{x_{j}}$ & $\begin{array}{l}\text { Accumulated interference from BSs of tier } j \text { at the } \\
\text { origin }\end{array}$ \\
\hline$\tilde{\Phi}_{i}=\left\{x_{i} \in \Phi_{i}: P_{i}\left\|x_{i}\right\|^{-\alpha} \geq \gamma\right\}$ & BSs of tier $i$ with detectable pilots \\
\hline$K_{P} \geq 1$ & $\begin{array}{l}\text { The maximum permissible measured pilots at the typ- } \\
\text { ical UE }\end{array}$ \\
\hline$n_{i}=0,1, \ldots, K_{P}$ & $\begin{array}{l}\text { The number of pilots shall be measured from tier } i \\
\left(\sum_{i} n_{i}=K_{P}\right)\end{array}$ \\
\hline$\overline{\Phi_{i} \subseteq \Phi_{i}}$ & Set of BSs of tier $i$ selected for CA \\
\hline$\overline{\bar{\tau}} \in[0,1]$ & Fraction of time slot designated for piloting and CA \\
\hline$R_{i}$ & $\begin{array}{l}\text { the prescribed data rate in tier } i \text {-if a typical UE } \\
\text { receives data rate lower than } R_{i} \text { it is experiencing } \\
\text { outage }\end{array}$ \\
\hline$\beta_{i}$ & $\begin{array}{l}\triangleq{ }_{2} R_{i}-1 \text {, the required SIR threshold to successfully } \\
\text { decode data under iCA model }\end{array}$ \\
\hline$\hat{\beta}_{i}$ & $\begin{array}{l}\triangleq_{2} \frac{R_{i}}{\left(1-\bar{\tau} K_{H}\right)^{+}}-1 \text {, the required SIR threshold } \\
\text { to successfully decode data under pCA model }\end{array}$ \\
\hline $\mathcal{P}_{C}\left(K_{P}\right)\left(\right.$ res. $\left.\mathcal{P}_{C i}\left(K_{P}\right)\right)$ & $\begin{array}{l}\text { Coverage probability (resp. coverage probability from } \\
\text { tier } i \text { ) under pCA model }\end{array}$ \\
\hline $\mathcal{P}_{C}^{\mathrm{iCA}}$ (resp. $\mathrm{ASE}^{\mathrm{iCA}}$ ) & $\begin{array}{l}\text { Coverage probability (resp. area spectral efficiency) } \\
\text { under iCA model }\end{array}$ \\
\hline$\gamma_{i}$ & $\triangleq \pi \lambda_{i}\left(\frac{\gamma}{P_{i}}\right)^{-\tilde{\alpha}}$ \\
\hline$\vartheta_{b_{i}}\left(\right.$ resp. $\left.\bar{\vartheta}_{n_{i}}\right)$ & 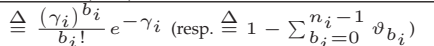 \\
\hline${ }^{f} \chi_{2 l}^{2}(\cdot)\left(\right.$ resp. $\mathrm{F}_{\chi_{2 l}^{2}}{ }^{(\cdot)}=1-\mathrm{F}_{\chi_{2 l}^{2}}{ }^{(\cdot))}$ & $\begin{array}{l}\text { Probability density function (pdf) (resp. cumulative } \\
\text { distribution function (cdf)) of a chi-squared random } \\
\text { variable (r.v.) with degree-of-freedoms (DoFs) } 2 l\end{array}$ \\
\hline
\end{tabular}

interference contribution of tier $j$ is a shot noise process, $I_{j}=\sum_{x_{j} \in \Phi_{j} / x_{0}} P_{j}\left\|x_{j}\right\|^{-\alpha} h_{x_{j}}$.

The cumulative distribution function (CDF), and the probability distribution function (pdf) of a chi-squared random variable (r.v.) with $2 l$ degrees of freedom (DoF) are denoted by $\mathrm{F}_{\chi_{2 l}^{2}}(t)$ and $\mathrm{f}_{\chi_{2 l}^{2}}(t)$, respectively.

Selecting the serving BS among all BSs, referred to as cell association (CA), plays an important role in the efficiency of a HetNet. The design of CA must account for a wide range of factors, such as pilot transmissions and its required resources, limitations in the pilot measurements, characteristics of the wireless medium, number of tiers, and their BSs' densities.

BSs frequently emit pilot signals to facilitate CA which is done in a time slotted fashion. In a time slot each UE utilizes the pilot signal to select its serving BS. The serving BS then transmits the intended data to the UE during that time slot. We define the permissible number of measured pilots, $K_{P} \geq 1$, as the maximum number of pilot signals that the typical UE can measure during one time slot. Let a time slot be $\tau$ seconds long and $\tau=$ $\tau_{\mathrm{D}}+K_{P} \tau_{\mathrm{T}}$, where $\tau_{\mathrm{D}}$ and $\tau_{\mathrm{T}}$ in a time slot are the time allocated to data transmission by the corresponding BS and measuring one pilot signal at the UEs, respectively. We also let $\bar{\tau}_{\mathrm{T}}=\tau_{\mathrm{T}} / \tau$ and $\bar{\tau}_{\mathrm{D}}=\tau_{\mathrm{D}} / \tau$ be the normalized sensing and transmission duration, respectively. So, $(1-$ $\left.\bar{\tau}_{T} K_{P}\right)^{+} \tau$ seconds of each time slot is available for data transmission, where $(y)^{+}=\max \{0, y\}$.

We will present a tractable analytical model for generic CA while considering its feasibility from the typical UE's 
perspective.

In the CA stage, the fading fluctuations are averaged out, and hence the received pilot signal power corresponding to BS $x_{i}$ at the typical UE is $P_{i}\left\|x_{i}\right\|^{-\alpha}$. Without loss of generality, we can assume that the power allocated to data transmission is equal to that of pilot transmission. It is in general straightforward to incorporate adaptive split of power between data and pilot transmission which is omitted for brevity. For the typical UE, BSs with detectable pilot signals constitute a set of candidate BSs, $\tilde{\Phi}$. The candidate set $\tilde{\Phi}$ is defined as $\tilde{\Phi}=\bigcup_{i=1}^{K}\left\{x_{i} \in \Phi_{i}, \forall i: P_{i}\left\|x_{i}\right\|^{-\alpha} \geq \gamma\right\}$, where $\gamma$ is the pilot detection threshold which we refer to as pilot sensitivity.

The larger the number of measured pilot signals, the higher the chance of finding the best possible BS for the typical UE to be associated with. In practice, however, the number of measured pilot signals is limited, because part of the time slot is required to be allocated for measuring each pilot signal. Therefore, increasing the number of measurements reduces the remaining part of the time slot available for data transmission.

Careful selection of $K_{P}$ plays an important role in the performance of CA. We also define $0 \leq n_{i} \leq K_{P}$ as the number of measured pilot signals of the BSs in tier $i$, so that $\sum_{i} n_{i}=K_{P}$, and $\tilde{\Phi}^{i} \subseteq \tilde{\Phi}$ as a collection of these $n_{i}$ BSs in tier $i$. Note that UE association with a BS located far away often results in frequent handovers during a data session/call, thus incurring extra signaling overheads. Therefore, it is preferable to associate a UE with a nearby BS with detectable pilots. This is consistent with the definition of $\tilde{\Phi}^{i}$ which consists of $n_{i}$ nearest BSs in tier $i$ to the typical UE.

Various CA schemes can be modeled based on the above parameters:

- Ideal CA (iCA) where pilot signals of all BSs in the network are measured by the UE and the one with the maximum SIR is then selected as the serving BS, i.e., $\gamma=0, \bar{\tau}_{P}=0$, and $K_{P}=|\Phi|$, where $|A|$ is the cardinality of set $A$. Throughout this paper we use iCA as the reference for comparison.

- Practical CA (pCA) where a BS in $\tilde{\Phi}$ with the maximum SIR value as in (1) is selected as the serving BS. Adjusting the model parameters in pCA provides new degrees of flexibility in the CA design for different HetNet scenarios.

Closely fitting the above model is the closest CA (cCA) whereby the closest BS across all tiers are chosen as the serving BS, regardless of its corresponding SIR, see, e.g., $[4,17,26]$.

\section{Design of Coverage-Optimal CA}

In this section, we focus on the design of CA for given HetNet settings, i.e., known $\left(\lambda_{i}, P_{i}, R_{i}\right)$ with the objective of maximizing network coverage probability. We will introduce a design process to obtain pCA parameters including $K_{P}$ and $n_{i}, \forall i$.
The design process developed in this section can be extended to cases with other performance metrics, e.g., spectral efficiency, or a combination of performance metrics.

\subsection{Coverage Probability}

The typical UE successfully receives the data transmitted by the serving BS $x_{i}$ if the corresponding scheduled data rate is greater than a pre-specified value $R_{i}>0$ (nat/sec/Hz). Here we note that the coverage probability is equal to the complementary cumulative distribution function (CCDF) of the SIR. Therefore, it is reasonable to consider SIR-based CA rules, i.e., CA rules which rely upon the SIR characteristics.

Some recent works on HetNet resource allocation e.g., see $[3,5,6,27,28]$ - show that disjoint treatment of cell association and resource allocation may degrade the achievable performance of the network, undermining the main motivation behind introducing HetNets. Therefore, cell association is obtained by solving an optimization problem where the objective function and constraints are formulated based on the physicallayer specifications, transmission policies, and scheduling procedures across tiers. The objective functions are often defined as a monotonically increasing function of SIR. Therefore, it is reasonable to consider maxSIR CA rule for modeling association. Furthermore, such works often overlook the impact of ICI and pilot measurements. We have addressed this issue, and also obtained a closed-form approximation based on simple CA policies which could not otherwise have been obtained.

'Coverage' for the typical UE means that there is at least one BS in the candidate set $\bigcup_{i=1}^{K} \tilde{\Phi}^{i}$ which can support the typical UE with the prescribed data rate $R_{i}$, i.e.,

$$
\mathcal{A}=\left\{\arg \max _{x_{i} \in \widetilde{\Phi}^{i}, \forall i}\left\{\log \left(1+\mathrm{SIR}_{x_{i}}\right) \geq \frac{R_{i}}{\left(1-\bar{\tau}_{P} K_{P}\right)^{+}}\right\}\right\},
$$

is not empty. For a given $K_{P}$, we then define the coverage probability as $\mathcal{P}_{C}\left(K_{P}\right)=\mathbb{P}\{\mathcal{A} \neq \emptyset\}$.

One may argue that the cCA method with biasing (e.g., range expansion method, e.g., $[4,26,29]$ ) can be considered as a CA alternative. Note that such biasing techniques mainly deal with technology selection, and merely attempts to offload traffic from one tier to another (for example, from Macro BSs to femto BSs). Therefore, they do not deal with the BS selection in each individual tier, and after selecting the technology (tier), cCA is used to associate the UE with the closest BS. We would also like to mention that it is quite straightforward to include biasing under the max-SIR CA rule in order to offload traffic across tiers.

In the rest of this paper we use the following notations: $\beta_{i} \triangleq e^{R_{i}}-1$ and $\hat{\beta}_{i} \triangleq e^{\frac{R_{i}}{\left(1-\bar{\tau}_{P} K_{P}\right)^{+}}}-1$. 


\subsection{Maximum Coverage pCA Design}

As shown in (2), increasing $K_{P}$ also increases $\hat{\beta}_{i}$. Therefore, although $\left|\bigcup_{i=1}^{K} \tilde{\Phi}^{i}\right|$ increases, i.e., a larger number of BSs in the candidate sets, the coverage probability may be decreased. For a given value of $K_{P}$, one can also adjust $\left(\lambda_{i}, P_{i}, R_{i}\right)$ in tier $i$, as well as $n_{i}=\left|\tilde{\Phi}^{i}\right|$ to maximize the HetNet's collective coverage probability. In what follows, we will formulate the CA design problem as an optimization problem with the objective of maximizing the coverage probability.

$$
\text { OP : } \max _{K_{P},\left\{n_{i} \geq 0\right\}} \mathcal{P}_{C}\left(K_{P}\right), \quad \text { s.t. } \sum_{i=1}^{K} n_{i}=K_{P} .
$$

In (3) the pCA parameters are designed based on the required coverage performance, which itself depends upon the average value of system parameter including $\left(\lambda_{i}, P_{i}, R_{i}\right), \forall i$. Therefore, the optimal $K_{P}$ and $\left\{n_{i}\right\}$ are required to be broadcasted to the UEs and kept updated if those average parameters change. For a given optimal pCA design, the obtained $K_{P}$ and $\left\{n_{i}\right\}$ are then fed into optimal resource allocation schemes within shorter time scales at the BSs/network. Such techniques have been investigated widely in the related literature, see, e.g., $[3,27]$, where they often assume a given cluster of BSs. Therefore, inter-cluster interference is overlooked and such techniques need to rely upon the availability of timely and accurate CSI among all BSs and UEs in the cluster. The optimization problem in (3) facilitate this process by providing an optimal set of candidate BSs to form the cluster and/or the initial power transmission of BSs.

Careful specification of the time scales in which OP and radio resource allocations operate, one can balance the signaling overheads required for acquiring CSI, on one hand, and the system complexity involved with the resource allocation, on the other.

In addition to the coverage probability, other network performance metrics, such as throughput and area spectral efficiency(ASE), or a combination thereof, could be considered as the optimization objective in (3). Examples of such an approach are given in $[4,7,26]$, where a utility function in most of them is defined based on the channels' CDF, which are often hard to obtain.

\subsection{Obtaining pCA's Optimal Parameters}

OP is a discrete optimization problem. The computational complexity of finding the optimal solution increases as $K_{P}$ and $K$ increase. We propose a greedy algorithm (Alg. 1) to find the optimal values of $K_{P}$ and $n_{i}$ s. This algorithm sequentially increases $K_{P}$ in each iteration such that $1 \leq K_{P} \leq\left\lfloor\frac{1}{\bar{\tau}_{P}}\right\rfloor$, where $\lfloor y\rfloor$ returns the largest integer number smaller than $y$. For the current value of $K_{P}$, the best partition resulting in the highest coverage probability is obtained through a greedy search. The algorithm then proceeds to the next iteration if the obtained coverage probability outperforms the one obtained in the previous iteration, $K_{P}-1$, else terminates.

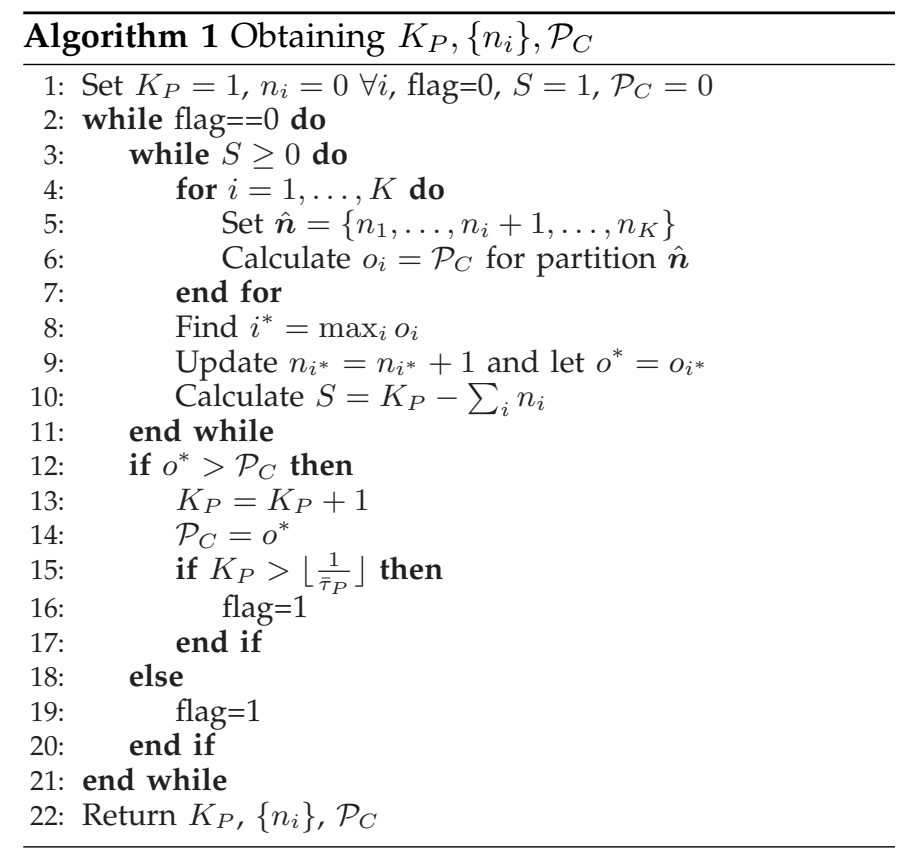

As shown in Alg. 1, to obtain the optimal pCA parameters, we need to obtain the coverage probability as a function of pCA parameters and network variable. We present this next.

\section{Coverage Performance Evaluation}

The following proposition approximates the coverage probability of a HetNet with a given pCA.

Proposition 1: The coverage probability of a $K$-tier HetNet, with $\left(\lambda_{i}, P_{i}, R_{i}\right), i=1, \ldots, K$, using pCA with $K_{P}$, and $\left\{n_{i}\right\}$ is

$$
\begin{gathered}
\mathcal{P}_{C} \lesssim \sum_{i=1}^{K} \psi_{i}, \\
\psi_{i}=\bar{\vartheta}_{n_{i}} \sum_{l=1}^{n_{i}} \varrho_{i}^{(l)}+\sum_{b_{i}=1}^{n_{i}-1} \vartheta_{b_{i}} \sum_{l=1}^{b_{i}} \varrho_{i}^{(l)},
\end{gathered}
$$

where ${ }_{i}^{(l)}$ refers to the $l^{\text {th }}$ closest BS to the typical UE in tier $i, \bar{\vartheta}_{n_{i}} \triangleq 1-\sum_{b_{i}=0}^{n_{i}-1} \vartheta_{b_{i}}$,

$$
\vartheta_{b_{i}} \triangleq \mathbb{P}\left\{\left|\hat{\Phi}^{i}\right|=b_{i}\right\}=\frac{\left(\gamma_{i}\right)^{b_{i}}}{b_{i} !} e^{-\gamma_{i}}
$$

$$
\begin{aligned}
& \gamma_{i} \triangleq \pi \lambda_{i}\left(\frac{\gamma}{P_{i}}\right)^{-\check{\alpha}} \text {, and } \\
& \varrho_{i}^{(l)} \triangleq \mathbb{P}\left\{\operatorname{SIR}_{x_{i}^{(l)}} \geq \hat{\beta}_{i}|| \tilde{\Phi}^{j} \mid=b_{i}, \forall j\right\} .
\end{aligned}
$$

Proof: See Appendix 1.

Remark 1: In Proposition 1, $\varrho_{i}^{(l)}$ defined in (6) is the conditional coverage probability of tier $i$ given the cardinality of its corresponding candidate BS set, $\left|\tilde{\Phi}^{i}\right|$.

As it is seen in Proposition 1, to obtain the approximate coverage probability for a given HetNet and pCA, one needs to obtain $\varrho_{i}^{(l)}$. As it is seen in (6), $\varrho_{i}^{(l)}$, is also a function of channel fading. In the following we evaluate $\varrho_{i}^{(l)}$ for Rayleigh and Nakagami fading channels. 


\subsection{Coverage Probability in Rayleigh Fading}

Having $\varrho_{i}^{(l)}$ one can obtain coverage probability using Propositions 1. The following Proposition provides a closed-form expression for $\varrho_{i}^{(l)}$ in Rayleigh fading environments.

Proposition 2: In a HetNet with pCA specified in Proposition $1, \varrho_{i}^{(l)}$ for Rayleigh fading environment is:

$$
\begin{aligned}
\varrho_{i}^{(l)} & =\int_{0}^{\gamma_{i}} \ldots \int_{0}^{\gamma_{i}} \frac{\mathrm{f}_{\chi_{2 l}^{2}}\left(x_{i_{l}}\right) e^{-\frac{x_{i_{l}}}{\hat{\kappa}_{i}}} d x_{i_{l}}}{\prod_{j} \prod_{l=1}^{b_{i}} \mathrm{~F}_{\chi_{2 l}^{2}}\left(\gamma_{j}\right)} \frac{\mathrm{f}_{\chi_{2 l^{\prime}}^{2}}\left(x_{i_{l^{\prime}}}\right) d x_{i_{l^{\prime}}}}{1+\hat{\beta}_{i}\left(\frac{x_{i_{l}}}{x_{i_{l^{\prime}}}}\right)^{\frac{\alpha}{2}}} \\
& \times \prod_{j \neq i} \int_{0}^{\gamma_{j}} \ldots \int_{0}^{\gamma_{j}} \prod_{l_{j}=1}^{b_{i}} \frac{\mathrm{f}_{\chi_{2 l_{j}}^{2}}\left(x_{j_{l_{j}}}\right) d x_{j_{l_{j}}}}{1+\hat{\beta}_{i} \frac{P_{j}}{P_{i}}\left(\frac{\lambda_{j}}{\lambda_{i}}\right)^{\frac{\alpha}{2}}\left(\frac{x_{i_{l}}}{x_{j_{l_{j}}}}\right)^{\frac{\alpha}{2}}}
\end{aligned}
$$

where $\hat{\kappa}_{i} \triangleq \frac{\lambda_{i}}{\kappa}\left(\frac{P_{i}}{\hat{\beta}_{i}}\right)^{\check{\alpha}}, \kappa \triangleq \frac{C(\alpha)}{\pi} \sum_{j} \kappa_{j}, C(\alpha) \triangleq$ $\check{\alpha} \pi^{2} \csc (\check{\alpha} \pi)$.

Proof: See Appendix 2.

Although the closed-from obtained in Proposition 2 articulates the impact of different system parameters, it is computationally complex. In the following propositions, we derive an approximation of $\varrho_{i}^{(l)}$ and a lower-bound which are both less computationally demanding than that of Proposition 2.

Proposition 3: An approximate of $\varrho_{i}^{(l)}$ is

$$
\varrho_{i}^{(l)} \approx \frac{1}{\mathrm{~F}_{\chi_{2 l}^{2}}\left(\gamma_{i}\right)} \int_{0}^{\gamma_{i}} \frac{e^{-\frac{x_{l}}{\hat{\kappa}_{i}}} \mathrm{f}_{\chi_{2 l}^{2}}\left(x_{l}\right) d x_{l}}{\left(1+\hat{\beta}_{i}\left(\frac{x_{l}}{\gamma_{i}}\right)^{\frac{\alpha}{2}}\right)^{\sum_{j=1}^{K} b_{j}-1}} d x_{l},
$$

Proof: See Appendix 3.

Proposition 4: A lower-bound on $\varrho_{i}^{(l)}$ is

$$
\varrho_{i}^{(l)} \geq \frac{1}{\mathrm{~F}_{\chi_{2 l}^{2}}\left(\gamma_{i}\right)} \frac{\Gamma\left(l, \gamma_{i}\left(1+\frac{1}{\hat{\kappa}_{i}}\right)\right)}{\left(1+\frac{1}{\hat{\kappa}_{i}}\right)^{l}},
$$

where $\Gamma(l, a)=1 / \Gamma(l) \int_{0}^{a} x^{l-1} e^{-x} d x$ (Incomplete Gamma function), and $\Gamma(l)=\int_{0}^{\infty} x^{l-1} e^{-x} d x$.

Proof: To obtain the lower-bound, we approximate (6) by

$$
\varrho_{i}^{(l)} \geq \mathbb{P}\left\{\operatorname{SIR}_{x_{i}^{(l)}} \geq \beta_{i} \mid\left\|x_{i}^{(l)}\right\|^{-\alpha} \geq \frac{\gamma}{P_{i}}\right\} .
$$

Following the same line of argument as in proof of Proportion 3 yields

$$
\varrho_{i}^{(l)} \geq \frac{1}{\mathrm{~F}_{\chi_{2 l}^{2}}\left(\gamma_{i}\right)} \int_{0}^{\gamma_{i}} e^{-\frac{x_{l}}{\kappa_{i}}} \mathrm{f}_{\chi_{2 l}^{2}}\left(x_{l}\right) d x_{l},
$$

which transfers to (9) using straightforward analytical derivations.

Compared to Propositions 2 and 3, the lower-bound in Proposition 4 has the lowest computational complexity. In this paper, we utilize the lower-bound in Proposition 4 , as it is easily obtainable to evaluate an approximation

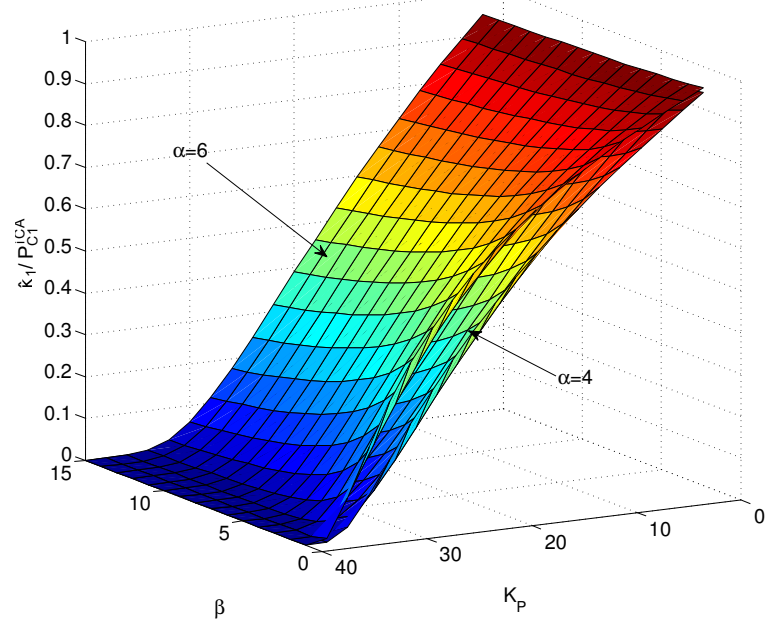

Fig. 1. $\frac{\hat{\mathrm{K}}_{1}}{\mathcal{P}_{C} \mathrm{iCA}}$ Vs. $\beta_{1}=\beta$, and $K_{P}$, for $\alpha=4,6$, where $\bar{\tau}_{P}=0.025$.

of the coverage probability and its interrelationships with other system parameters. As it is seen in Propositions 3 and 4 , the bound/approximation of $\varrho_{i}^{(l)}$ is a function of various parameters and it is not straightforward to obtain equality conditions.

\subsection{1 iCA Coverage Probability with Rayleigh Fading}

The coverage probability of tier $i$ with iCA, $\mathcal{P}_{C i}^{\mathrm{iCA}}=$ $\mathbb{P}\left\{\max _{x_{i} \in \Phi_{i}} \operatorname{SIR}_{x_{i}} \geq \beta_{i}\right\}$, is derived in [7]:

$$
\mathcal{P}_{C i}^{\mathrm{iCA}}=\frac{\pi \lambda_{i} P_{i}^{\check{\alpha}} \beta_{i}^{-\check{\alpha}}}{C(\alpha) \sum_{j} \lambda_{j} P_{j}^{\check{\alpha}}}
$$

In iCA, UEs are able to measure all the transmitted pilots, and select the best BS from the candidate set $\tilde{\Phi}^{i}$ which is the same as $\Phi^{i}, \forall i$ with the assumption of $\bar{\tau}_{P}=$ 0 . Therefore, the coverage probability in (11) is larger than, or equal to the case of pCA.

Starting from the definition of $\hat{\kappa}_{i}$ in Proposition 2, it is straightforward to show that

$$
\hat{\kappa}_{i}=\left(\frac{\beta_{i}}{\hat{\beta}_{i}}\right)^{\check{\alpha}} \mathcal{P}_{C i}^{\mathrm{iCA}}=\frac{\beta_{i}^{\check{\alpha}} \mathcal{P}_{C i}^{\mathrm{iCA}}}{\left(\left(1+\beta_{i}\right)^{\frac{1}{\left(1-\bar{\tau}_{P} K_{P}\right)^{+}}}-1\right)^{\check{\alpha}}} .
$$

As shown in (12), $\hat{\kappa}_{i}$ is directly related to the coverage probability offered by tier $i$, where iCA is adopted. It is also seen that (12) depends solely on parameter $K_{P}$, and is in fact independent of $\left\{n_{i}\right\}$. In general, $\frac{\beta_{i}}{\bar{\beta}_{i}} \leq 1$, and hence for larger values of the path-loss exponent, $\alpha$, $\hat{\kappa}_{i} \rightarrow \mathcal{P}_{C i}^{\mathrm{iCA}}$.

Fig. 1 plots $\frac{\hat{\kappa}_{1}}{\mathcal{P}_{C}^{\text {iCA }}}$ versus $K_{P}$ and $\beta$. The figure shows that increasing $\beta \geq 1$ results in a slight reduction of $\frac{\hat{k}_{1}}{\mathcal{P}_{C}^{\text {iCA }}}$. Also, by increasing $K_{P}, \frac{\hat{\kappa}_{1}}{\mathcal{P}_{C}^{\text {idA }}}$ is steadily reduced to zero.

The relationship between the coverage probability of tier $i$ in iCA and parameter $\hat{\kappa}_{i}$ in (12), as well as the results in Propositions 1-4, suggests that the coverage probability in a HetNet with pCA might be related to the coverage probability in a HetNet with iCA. Therefore, one may consider designing/analyzing the network 
based on the iCA model and then make a corresponding adjustment to incorporate the impacts of pCA. However, the issue is whether a HetNet with pCA responds to densification in the same way as in the iCA. We investigate this issue in Section 5.

\subsection{Coverage Probability in Nakagami Fading}

To obtain the coverage probability in a Nakagami fading environment, similarly to Section 4.1 , we obtain $\varrho_{i}^{(l)}$ based on the method of Proposition 4. Nakagami fading is an accurate model for the channel fading in post-processing SIR in some MIMO communication paradigms such as eigen-beamforming, and/or spacedivision multiple access (SDMA), see, e.g., [8,30-32].

In a Nakagami fading environment, the channel power gain in tier $i$ is distributed according to a Chi-square distribution with $2 M_{i}$ degrees of freedom (DoFs). This model allows $M_{i} \mathbf{s}$ to have different values across tiers depending on the wireless environment/technology (e.g., indoor/outdoor or urban/suburban, MIMO, etc.). For example, in a MIMO system, Nakagami parameters are obtained based on the number of transmit antennas, number of served UEs, and beamforming technique, see, e.g., [8,30-32]. Nakagami-type fading models are also used to model MIMO multiplexing maximum ratio combining (MRC), MIMO multiplexing ZFBF, MIMO orthogonal space time block coding (OSTBC), and MIMO singular value decomposition (SVD), see, e.g., [33-37] ${ }^{1}$.

Proposition 5: In a HetNet adopting pCA, $\varrho_{i}^{(l)}$ for a Nakagami fading environment is lower-bounded as

$$
\begin{gathered}
\varrho_{i}^{(l)} \geq \frac{1}{\mathrm{~F}_{\chi_{2 l}^{2}}\left(\gamma_{i}\right)} \frac{\Gamma\left(l, \gamma_{i}\left(1+\frac{1}{\hat{\kappa}_{i}\left(\left\{M_{j}\right\}\right)}\right)\right)}{\left(1+\frac{1}{\hat{\kappa}_{i}\left(\left\{M_{j}\right\}\right)}\right)^{l}}+ \\
\sum_{m=1}^{M_{i}-1} \sum_{n=1}^{m} \frac{(-1)^{m} \phi_{n}^{m} \Gamma(n+l) \Gamma\left(n+l, \gamma_{i}\left(1+\frac{1}{\hat{\kappa}_{i}\left(\left\{M_{j}\right\}\right)}\right)\right)}{n ! m ! \mathrm{F}_{\chi_{2 l}^{2}}\left(\gamma_{i}\right)\left(1+\frac{1}{\hat{\kappa}_{i}\left(\left\{M_{j}\right\}\right)}\right)^{n+l}},
\end{gathered}
$$

1. For instance, consider a HetNet system where zero-forcing beam-forming (ZFBF) is employed in all tiers (i.e., space division multiple access (SDMA)). In this system BSs in tier 1 (tier 2) have $N_{1}$ $\left(N_{2}\right)$ transmit antennas and serve $1 \leq U_{1}<N_{1}+1\left(1 \leq U_{2}<N_{2}+1\right.$ ) UEs in the downlink. For such a system, it is shown that the fading model of the intended signal in tier 1 (tier 2) is Nakagami $\left(N_{1}-U_{1}+1,1\right)$ (Nakagami $\left.\left(N_{2}-U_{2}+1,1\right)\right)$ (see, $\left.[8,31]\right)$. It is further shown in same references that the interfering gains can be modelled by Nakagami $\left(U_{1}, 1\right)$ in tier 1 and Nakagami $\left(U_{2}, 1\right)$ in tier 2 . Now assume that tier 1 serves as $N_{1}$ UEs. In this case, the intended signal for each served UE experiences Rayleigh fading, while the interfering signals from the BSs in tire 1 (tier 2) experience Nakagami $\left(N_{1}, 1\right)$ (Nakagami $\left(U_{2}, 1\right)$ ). As a result, the average received power of the intended signal from a BS of tier 1 reduces to 1, while the average power of interfering signals is either $N_{1} \gg 1$ (from BSs of tier 1) or $U_{2}$ (from BSs of tier 2). Therefore, although tier 1 serves as many UEs as it can, each may suffer from weakened intending signal power, thus a lower coverage performance. In practice however, one may adopt an adaptive balancing mechanism which adjusts the maximum number of served UEs, based on the desired coverage performance. where

$$
\begin{gathered}
\phi_{n}^{m} \triangleq \sum_{p=1}^{n}(-1)^{p}\left(\begin{array}{l}
n \\
p
\end{array}\right) \frac{\Gamma(\check{\alpha} p+1)}{\Gamma(\check{\alpha} p-m+1)}, \\
\hat{\kappa}_{i}\left(\left\{M_{j}\right\}\right) \triangleq \frac{\lambda_{i}}{\kappa\left(\left\{M_{j}\right\}\right)}\left(\frac{P_{i}}{\hat{\beta}_{i}}\right)^{\check{\alpha}} \text {, and } \\
\kappa\left(\left\{M_{j}\right\}\right) \triangleq \Gamma(1-\check{\alpha}) \sum_{j} \lambda_{j} P_{j}^{\check{\alpha}} \frac{\Gamma\left(\check{\alpha}+M_{j}\right)}{\Gamma\left(M_{j}\right)} .
\end{gathered}
$$

Proof: See Appendix 4.

In the lower bound provided by Proposition 5, the impact of interference is explicitly captured through $\kappa\left(\left\{M_{j}\right\}\right)$ which proportionally increases with BSs' densities. One may also note the resemblance between Propositions 4 and 5. In fact, replacing $\hat{\kappa}_{i}$ in Proposition 4 with $\hat{\kappa}_{i}\left(\left\{M_{j}\right\}\right)$ is the same as the first term of (13). The second term in (13) is also a summation of scaled and adjusted versions of the first term.

\subsection{1 iCA Coverage Probability with Nakagami Fading}

Following the same line of argument as in the proof of Proposition 5, the coverage probability of tier $i$ with iCA in a Nakagami fading environment, $\mathcal{P}_{C}^{\mathrm{iCA} A \mathrm{Nak}]}$ is

$$
\begin{gathered}
\mathcal{P}_{C[N a k]}^{\mathrm{iCA}}=\mathbb{P}\left\{\max _{x_{i} \in \Phi_{i}} \operatorname{SIR}_{x_{i}} \geq \beta_{i}\right\} \\
=2 \pi \lambda_{i} \int_{0}^{\infty} x_{i} \int_{0}^{\infty} \mathcal{L}_{\bar{F}_{h}}^{-1}(t) \prod_{j=1}^{K} \mathcal{L}_{I_{j}}\left(t \frac{\beta_{i} x_{i}^{\alpha}}{P_{i}}\right) d t d x_{i} \\
=2 \pi \lambda_{i} \int_{0}^{\infty} x_{i} \int_{0}^{\infty} \mathcal{L}_{\bar{F}_{h}}^{-1}(t) e^{-\left(t \frac{\beta_{i}}{P_{i}}\right)^{\check{\alpha}} \kappa\left(\left\{M_{j}\right\}\right) x_{i}^{2}} d t d x_{i} \\
=2 \pi \lambda_{i} \int_{0}^{\infty} \mathcal{L}_{\bar{F}_{h}}^{-1}(t) \int_{0}^{\infty} x_{i} e^{-\left(t \frac{\beta_{i}}{P_{i}}\right)^{\check{\alpha}} \kappa\left(\left\{M_{j}\right\}\right) x_{i}^{2}} d x_{i} d t \\
=\frac{\pi \lambda_{i}}{\kappa\left(\left\{M_{j}\right\}\right)} \int_{0}^{\infty} \frac{\mathcal{L}_{\bar{F}_{h}}^{-1}(t)}{\left(t \frac{\beta_{i}}{P_{i}}\right)^{\check{\alpha}}} d t=\left.\frac{\left(\frac{P_{i}}{\beta_{i}}\right)^{\check{\alpha}}}{\kappa\left(\left\{M_{j}\right\}\right)} \sum_{m=0}^{M_{i}-1} \frac{(-1)^{m}}{m !} \frac{d^{m}}{d t^{m}} t^{-\check{\alpha}}\right|_{t=1} .
\end{gathered}
$$

Applying the definition of $\hat{\kappa}_{i}\left(\left\{M_{j}\right\}\right),(15)$ is reduced to:

$$
\begin{aligned}
\mathcal{P}_{C i[N a k]}^{\mathrm{iCA}}= & \left(\frac{\beta_{i}}{\hat{\beta}_{i}}\right)^{-\check{\alpha}} \hat{\kappa}_{i}\left(\left\{M_{j}\right\}\right) \sum_{m=0}^{M_{i}-1} \frac{\Gamma(\check{\alpha}+m)}{\Gamma(\check{\alpha}) \Gamma(m+1)} \\
= & \frac{\lambda_{i}\left(\frac{P_{i}}{\beta_{i}}\right) \sum_{m=0}^{\check{\alpha}} \frac{M_{i}-1}{\Gamma(\check{\alpha}+m)}}{\pi \csc (\check{\alpha} \pi) \sum_{j=1}^{K} \lambda_{j} P_{j} \check{\alpha} \frac{\Gamma\left(\check{\alpha}+M_{j}\right)}{\Gamma\left(M_{j}\right)}} .
\end{aligned}
$$

The coverage probability given in (16) is significantly simpler than the existing results on the coverage probability of Nakagami fading environments, see, e.g., [8, 30,40,41]. This is because max-SIR CA implies that the interferers might be even closer to the typical UE than that of the serving BS (as there is no guarantee that the closest BS can provide the highest SIR). Therefore, compared to CCA, in our analysis we do not 
require to consider guard zone around the typical UE to calculate the Laplace transform of the interference. Under cCA rule the corresponding Interference Laplace transform is presented through complex integral functions. In such cases separating variable $s$ from the other variables is not straightforward. This then results in often intractable higher-order differentiations due to Nakagami-type fading.

Comparing the coverage probability of a Rayleigh environment in (12) and (16), one can find that in the case of Nakagami fading, the signal strength is multiplied by $\check{\alpha} \sum_{m=0}^{M_{i}-1} \frac{\Gamma(\check{\alpha}+m)}{m \Gamma(m)}$, while the strength of the interference caused by tier $j$ is multiplied by $\frac{\Gamma\left(\check{\alpha}+M_{j}\right)}{\Gamma\left(M_{j}\right)}$. It is, however, not directly seen in (16) which of these mentioned effects is dominant.

Exploiting the stochastic ordering results in [8], one can show that the coverage probability of Rayleigh fading outperforms that of Nakagami fading. This can be also understood by noting that when network is not dense, compared to the Rayleigh fading, in a Nakagami fading channel with $M_{i} \gg 1$, the fading fluctuations are (partially) dampened. Therefore, the signal power is weakened by the increased ICI. On the other hand, when the network is densified, both signal and ICI are adequately powerful so that Nakagami fading with $M_{i}>1$ could not make any tangible effects of the SIR distribution, compared to that with Rayleigh fading.

Fig. 2 plots the coverage probability of a two-tier HetNet with iCA versus $M_{1}$ and $M_{2}$ for different tier 2 densities $\lambda_{2}=10^{-3}$ and $\lambda_{2}=10^{-2}$. As seen in both HetNets with different densities in a Rayleigh environment $\left(M_{1}=M_{2}=1\right)$, the coverage probability is greater than that of a Nakagami environment $\left(M_{1}>1\right.$ and $\left.M_{2}>1\right)$. Also, the rate of reduction in $\mathcal{P}_{C[N a k]}^{\mathrm{iCA}}$ due to an increase of $M_{2}$ is larger than that of $M_{1}$, which is mainly because $\lambda_{2}>\lambda_{1}$. It is further seen that for a highly dense tier 2, $\lambda_{2}=10^{-2}$, the coverage probability is not a function of $M_{1}$.

\section{IMPACT OF DENSIFICATION}

Highly dense HetNets are used in wireless networks to meet high wireless connectivity demands and are also seen as an integral part of future 5G networks [1]. It is therefore of utmost importance to understand the impact of CA on the coverage performance of dense HetNets. We now investigate the following two important questions in dense HetNets:

- How relevant is pilot sensitivity to the CA coverage performance?

- Is HetNet scale invariancy sustainable where pCA is adopted?

For brevity, we focus our analysis on Rayleigh fading, and assume that in a dense HetNet $\lambda_{i}, \tilde{\Phi}_{i}$ is large enough for all $i$. A HetNet is referred to as a dense HetNet if, $\lambda_{i} \geq \frac{1}{\pi}\left(\frac{\gamma}{P_{i}}\right)^{\alpha}, \forall i$.

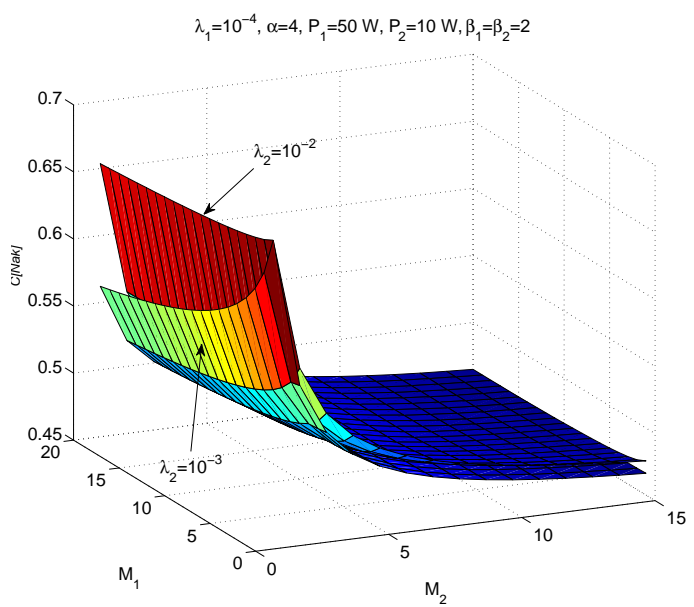

Fig. 2. The coverage probability of iCA vs. $M_{1}$ and $M_{2}$ with Nakagami fading, for $\lambda_{2}=10^{-3}$ and $\lambda_{2}=10^{-2}$, where $\alpha=4, P_{1}=50 \mathrm{~W}, P_{2}=10 \mathrm{~W}$ and $\beta_{1}=\beta_{2}=2$.

\subsection{Pilot Neutrality}

To investigate the impact of pilot sensitivity densification on the coverage probability as a benchmark, we define pilot neutrality as follows.

Definition Pilot Neutrality: A pCA is pilot-neutral if $\gamma=0$.

In a pilot-neutral pCA model, all the BSs in the coverage area are in the candidate set.

Proposition 6: In a dense HetNet with pilot-neutral pCA, the coverage probability is:

$$
\mathcal{P}_{C} \leq \sum_{i} \hat{\kappa}_{i}\left(1-\left(1+\frac{1}{\hat{\kappa}_{i}}\right)^{-n_{i}}\right) .
$$

Proof: Given $\gamma=0$, then as given in Proposition $1, \vartheta_{b_{i}}=$ 0 and $\bar{\vartheta}_{n_{i}}=1$. Therefore,

$$
\begin{gathered}
\mathcal{P}_{C} \leq \sum_{i} \sum_{l=1}^{n_{i}} \mathbb{E}_{x_{i}^{(l)}} \mathbb{P}\left\{\operatorname{SIR}_{x_{i}^{(l)}} \geq \hat{\beta}_{i} \mid x_{i}^{(l)}\right\} \\
=\sum_{i} \sum_{l=1}^{n_{i}} \int_{0}^{\infty} f_{\chi_{2 l}^{2}}(x) e^{-\frac{x}{\kappa_{i}}} d x=\sum_{i} \sum_{l=1}^{n_{i}}\left(1+\frac{1}{\hat{\kappa}_{i}}\right)^{-l},
\end{gathered}
$$

which completes the proof.

In a generic pCA model, the received power of the pilot signals associated with the BSs in the candidate set must be stronger than the pilot sensitivity, $\gamma$. In a dense HetNet setting however, there might be more than $n_{i}$ BSs in each tier $i$ fulfilling the pilot sensitivity requirement.

We note that in (17) $\mathcal{P}_{C} i$ is a function of $\left\{n_{i}\right\}, \hat{\kappa}_{i}$, where $\hat{\kappa}_{i}$ is also related to the coverage probability in a HetNet with iCA, (12), $\mathcal{P}_{C i}^{\mathrm{iCA}}: \hat{\kappa}_{i}=\left(\frac{\beta_{i}}{\hat{\beta}_{i}}\right)^{\check{\alpha}} \mathcal{P}_{C i}^{\mathrm{iCA}}$. Therefore, the coverage probability of tier $i$ in a scale invariant HetNet, (17) can be written as

$$
\mathcal{P}_{C i} \leq\left(\frac{\beta_{i}}{\hat{\beta}_{i}}\right)^{\check{\alpha}} \mathcal{P}_{C i}^{\mathrm{iCA}}\left(1-\frac{\left(\left(\frac{\beta_{i}}{\hat{\beta}_{i}}\right)^{\check{\alpha}} \mathcal{P}_{C i}^{\mathrm{i} C A}\right)^{n_{i}}}{\left(1+\left(\frac{\beta_{i}}{\bar{\beta}_{i}}\right)^{\check{\alpha}} \mathcal{P}_{C i}^{\mathrm{iCA}}\right)^{n_{i}}}\right) .
$$


In (18), $\left(\frac{\beta_{i}}{\hat{\beta}_{i}}\right)^{\check{\alpha}} \leq 1$, where $\frac{\beta_{i}}{\hat{\beta}_{i}}$ depends only on $K_{P}$ as $\hat{\beta}_{i}=\left(1+\beta_{i}\right)^{\frac{1}{\left(1-\bar{\tau}_{P} K_{P}\right)^{+}}}-1$. Therefore, (18) shows that the coverage probability of a HetNet degrades compared to the pilot-neutral case, even if iCA is adopted. One may further reduce the degree of degradation by carefully partitioning the candidate set as it is also a function of $\left\{n_{i}\right\}$.

Proposition 7: If $\lambda_{i} \geq \frac{1}{\pi}\left(\frac{\gamma}{P_{i}}\right)^{\check{\alpha}}, \forall i$, the coverage probability of a dense HetNet with a generic pCA can be approximated by the coverage probability of the same network with pilot-neutral pCA.

Proof: See Appendix 5.

Proposition 8: In a HetNet with pilot-neutral pCA the coverage probability is approximated by

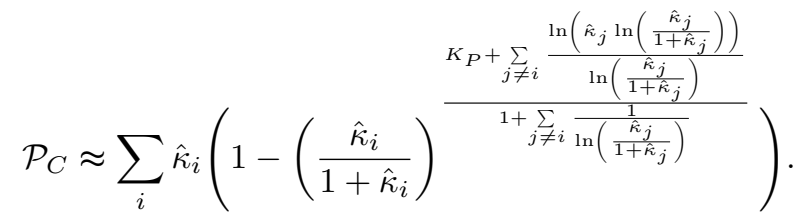

Proof: See Appendix 6.

Proposition 8 provides the coverage probability in a closed-form expression which depends only on $K_{P}$ and parameters $\hat{\kappa}_{i} \mathrm{~s}$ assuming that the network is dense.

\subsection{Scale Invariance}

In cellular networks, scale invariancy means that increasing the density of the BSs does not affect the coverage probability while causing a proportional growth in the Area spectral efficiency (ASE), see, e.g., $[1,7,42]$. Scale invariance is one of the main benefits of densification in cellular networks as it can increase the capacity without compromising the coverage.

ASE is often considered as a metric to characterize the capacity of a cellular network. The ASE of a HetNet employing iCA is defined as

$$
\mathrm{ASE}^{\mathrm{iCA}} \triangleq \sum_{i} \lambda_{i} \mathcal{P}_{C i}^{\mathrm{iCA}} R_{i}=\frac{\frac{\pi}{C(\alpha)} R}{\left(e^{R}-1\right)^{\check{\alpha}}} \frac{\sum_{j} \lambda_{j}^{2} P_{j}^{\check{\alpha}}}{\sum_{j} \lambda_{j} P_{j}^{\check{\alpha}}},
$$

and is measured in nat $/ \mathrm{sec} / \mathrm{Hz} / \mathrm{m}^{2}$. Here we assume that $R_{i}=R$, or equivalently $\beta_{i}=\beta$, for all $i$. In a HetNet with iCA, the coverage probability in (11) is simplified to $\mathcal{P}_{C}^{\mathrm{iCA}}=\frac{\pi}{C(\alpha) \beta^{\tilde{\alpha}}}$. Increasing the BSs' densities does not affect the coverage probability. We also show that increasing the BSs'densities causes a proportional growth in the ASE of such systems.

Proposition 9: In a HetNet with iCA and $\beta_{i}=\beta, \forall i$, ASE grows with the increasing of $\min _{i} \lambda_{i}$ via

$$
\operatorname{ASE}^{\mathrm{iCA}}<C^{*}(\alpha) \min _{i} \lambda_{i}
$$

where $C^{*}(\alpha)$ only depends on $\alpha$.

Proof: To increase ASE by increasing $\lambda_{i}$, we need to have $\frac{\partial}{\partial \lambda_{i}} \mathrm{ASE}^{\mathrm{iCA}}>0, \forall i$. By differentiating (20) with respect to $\lambda_{i}$, we can see that $\frac{\partial}{\partial \lambda_{i}} \mathrm{ASE}^{\mathrm{iCA}}>0$ holds if $2 \lambda_{i} \sum_{j} \lambda_{j} P_{j}^{\check{\alpha}}>\sum_{j} \lambda_{j}^{2} P_{j}^{\check{\alpha}} \forall i$. Using (20), this can equivalently be written as $\mathrm{ASE}^{\mathrm{iCA}}<\frac{2 \pi R}{C(\alpha)\left(e^{R}-1\right)^{\dot{\alpha}}} \lambda_{i}, \forall i$, and thus

$$
\mathrm{ASE}^{\mathrm{iCA}}<\frac{2 \pi R}{C(\alpha)\left(e^{R}-1\right)^{\check{\alpha}}} \min _{i} \lambda_{i} .
$$

We then take a derivative of the right hand side of the inequality in (21) with respect to $R$, and set the resultant equal to zero, i.e., $e^{-R}=1-\check{\alpha} R$. Note that the solution of this equation, $R^{*}$, depends only on $\alpha$. Substituting $R^{*}$ in (21) and introducing $C^{*}(\alpha)=\frac{2 \pi\left(R^{*}\right)^{1-\check{\alpha}} e^{-\check{\alpha} R^{*}}}{C(\alpha) \check{\alpha}^{\check{\alpha}}}$, which depends only on $\alpha$, finalizes the proof .

The following proposition investigates scale invariancy of a HetNet with pCA.

Proposition 10: A HetNet with pCA and $\beta_{i}=\beta, \forall i$, is not scale-invariant.

Proof: For scale invariancy, we need to check whether $\frac{\partial}{\partial \lambda_{i}} \mathcal{P}_{C}=0$,

$$
\frac{\partial}{\partial \lambda_{i}} \mathcal{P}_{C}=\frac{\partial}{\partial \lambda_{i}} \sum_{j=1}^{K} \hat{\kappa}_{j}-\sum_{j=1}^{K} \frac{\partial \epsilon\left(n_{j}\right)}{\partial \hat{\kappa}_{j}} \frac{\partial \hat{\kappa}_{j}}{\partial \lambda_{i}},
$$

where $\epsilon\left(n_{i}\right) \triangleq \hat{\kappa}_{i}\left(1+\frac{1}{\hat{\kappa}_{i}}\right)^{-n_{i}}$, and $K$ is the number of tiers. The first term in (22) is zero as $\sum_{j=1}^{K} \hat{\kappa}_{j}$ is independent of densities, $\lambda_{i}$, as $\beta_{i}=\beta, \forall i$. Regarding the second term in (22) we further show that

$$
\begin{gathered}
\frac{C(\alpha)}{\pi} \sum_{j=1}^{K} \frac{\partial \epsilon\left(n_{j}\right)}{\partial \hat{\kappa}_{j}} \frac{\partial \hat{\kappa}_{j}}{\partial \lambda_{i}}=\frac{\left(1+\frac{n_{i}}{1+\hat{\kappa}_{i}}\right)}{\left(\frac{\hat{\kappa}_{i}}{1+\hat{\kappa}_{i}}\right)-n_{i}} \frac{\hat{\kappa}_{i}}{\lambda_{i}} \frac{\sum_{k \neq i} \lambda_{k} P_{k}^{\check{\alpha}}}{\sum_{k} \lambda_{k} P_{k}^{\check{\alpha}}} \\
-\sum_{j \neq i} \frac{\left(1+\frac{n_{j}}{1+\hat{\kappa}_{j}}\right)}{\left(\frac{\hat{\kappa}_{j}}{1+\hat{\kappa}_{j}}\right)-n_{j}} \sum_{k} \hat{\kappa}_{j} P_{i}^{\check{\alpha}} P_{k}^{\check{\alpha}} \\
=\frac{\left(1+\frac{n_{i}}{1+\hat{\kappa}_{i}}\right)}{\left(\frac{\hat{\kappa}_{i}}{1+\hat{\kappa}_{i}}\right)^{-n_{i}}} \frac{\hat{\kappa}_{i}}{\lambda_{i}}-\frac{P_{i}^{\check{\alpha}}}{\sum_{k} \lambda_{k} P_{k}^{\check{\alpha}}} \sum_{j} \frac{\left(1+\frac{n_{j}}{1+\hat{\kappa}_{j}}\right)}{\left(\frac{\hat{\kappa}_{j}}{1+\hat{\kappa}_{j}}\right)^{-n_{j}}} \hat{\kappa}_{j} \\
=\frac{\left(1+\frac{n_{i}}{1+\kappa^{\prime}}\right)}{\left(\frac{\kappa^{\prime}}{1+\kappa^{\prime}}\right) n^{-n_{i}}} \frac{\kappa^{\prime}}{\lambda_{i}}-\frac{2 \kappa^{\prime}}{\lambda_{i}} \sum_{j \neq i} \frac{\left(1+\frac{n_{j}}{1+\kappa^{\prime}}\right)}{\left(\frac{\kappa^{\prime}}{1+\kappa^{\prime}}\right)^{-n_{j}}},
\end{gathered}
$$

where $\kappa^{\prime}=\frac{\pi}{C(\alpha) \hat{\beta}^{\tilde{\alpha}}}$ and in the last step, we note that $\hat{\kappa}_{i}$ is index-independent. We then set (23) equal to zero, and sum up the resultants on $i$ :

$$
\sum_{i} \frac{\left(1+\frac{n_{i}}{1+\kappa^{\prime}}\right)}{\left(\frac{\kappa^{\prime}}{1+\kappa^{\prime}}\right)^{-n_{i}}}=2(K-1) \sum_{i} \frac{\left(1+\frac{n_{i}}{1+\kappa^{\prime}}\right)}{\left(\frac{\kappa^{\prime}}{1+\kappa^{\prime}}\right)^{-n_{i}}},
$$

which is only valid if $K=1.5$, where $K$ is the number of tiers. This shows that coverage probability is not independent of $\lambda_{i}$, and thus scale-invariancy is not valid where pCA is adopted.

Proposition 10 indicates that the scale-invariancy of a HetNet with iCA is not sustained where pCA is adopted. Scale-invariancy of HetNets with cCA is also shown to be compromised where path-loss model deviates from the standard form $\|x\|^{-\alpha}$, [42-44]. Proposition 10 further shows that even with unbounded path loss, scale invariancy of HetNets with cCA is not maintained due to the pilot measurements required in the cell association. 


\section{Simulation Results}

Our main objectives in this section are to examine the accuracy of our analytical results, to evaluate the performance of Alg. 1, and to understand the effect of optimal pCA design on the coverage performance of HetNets. We simulate a two-tier HetNet, $K=2$, where macro BSs in the first tier have a hight transmission power of $P_{1}=50 \mathrm{~W}$. The second tier consists of femtocells covered by BSs with a much lower transmission power of $P_{2}=10 \mathrm{~W}$. The path-loss exponent is $\alpha=4$ and $\bar{\tau}_{P}=0.025$. The BSs in each tier are randomly distributed within in a disk with radius 10,000 units according to the corresponding tier densities. The Monte Carlo technique is adopted and we analyzed the results of 40,000 simulation snapshots.

\subsection{Accuracy of Analysis}

\subsubsection{Rayleigh Fading}

We now assess the accuracy of the coverage probability given in Propositions 1-4 for a given $K_{P}$. We also set $K=2, P_{1}=50 \mathrm{~W}, P_{2}=10 \mathrm{~W}, \alpha=4$, and $\bar{\tau}_{P}=0.025$.

Fig. 3 plots coverage probability versus $K_{P}$ for $R_{2}=$ 3.5 and $R_{2}=2$. As expected, by increasing $R_{2}$, it becomes harder to meet the required data rate and the coverage probability is consequently reduced. The results in Fig. 3 also indicate that the approximations derived in Propositions 3 and 4 are very close to the actual coverage probability.

It is further seen that by increasing $K_{P}$, coverage performance is improved to a maximum level before it starts declining. The initial improvement of $\mathcal{P}_{C}$ is because of availability of more options (BSs to be associated with) in the candidate set. By increasing $K_{P}$ further, the portion of time-slot allocated to pilot measurement becomes larger, thus making it harder to meet the required transmission rate. Accordingly, this reduces the coverage probability. This effect is often overlooked in the previous studies, see, e.g., Theorem III.1 in [24]. Fig. 3 also highlights the gap between the coverage performance in two HetNets with pCA and iCA. Interestingly, for a small candidate set, $2 \leq K_{P} \leq 6$, this performance difference is relatively small.

Impact of BSs' densities on coverage performance is investigated in Fig. 4, where we set $K_{P}=6$, and $n_{1}=n_{2}=3$. Fig. 4 shows that compared to Proposition 3 , Proposition 4 provides a more accurate approximation of coverage probability. The gap between the coverage performance approximation given in Proposition 3 and that of the simulation is higher for lower density of BSs. In contrast, the approximation by Proposition 4 preserves its accuracy by changing the BS densities. There is a rather large gap between the coverage performance under pCA and iCA models, where $\lambda_{1}=10^{-4}$, and $\lambda_{2} \leq 10^{-3}$. The difference is, however, decreased by increasing $\lambda_{2}$.

In this example, we set $\gamma=10^{-5}$, which is rather a large threshold. Therefore, in cases where the density of
BSs in tiers are small, it is unlikely to find the proper serving BS even if $K_{P}=6$. By increasing $\lambda_{1}$ to $10^{-2}$, the coverage probability of $\mathrm{pCA}$ and that of iCA are very close.

One can also see that by densifing tier 1 , the coverage probability of the system with iCA is reduced by increasing $\lambda_{2}$, while it is improved in pCA. This observation is consistent with our assertion of the critical importance of practical CA models.

The coverage probability versus pilot sensitivity, $\gamma$ is plotted in Fig. 5. The approximation given by Proposition 4 is shown to closely follow the simulations as opposed to that of in Proposition 3 whose accuracy declines by increasing $\gamma$. For $\lambda_{2}=10^{-4}$, the difference between the coverage performance in $\mathrm{pCA}$ and iCA is further increased by increasing $\gamma$. Pilot-neutrality is observed when tier 2 is dense, i.e., $\lambda_{2}=10^{-2}$ as the coverage performance is not affected by varying $\gamma$.

Propositions 3, and 4 respectively provide an approximation, and a lower bound for $\varrho_{i}^{(l)}$. These are then used in Proposition 1 to obtain the coverage probability in Figs. 3-5. This is also shown in Figs. 3-5 that the coverage approximation obtained based on Propositions 3 and 4, demonstrate different behavior in different scenarios. However, one can see in Figs. 3-5 that simulation results are often closely followed by the coverage probability obtained based on Proposition 4. This might be because Proposition 1 provides an upper-bound to the coverage probability while Proposition 4 provides a lower-bound on $\varrho_{i}^{(l)}$.

\subsubsection{Nakagami Fading}

For a system with Nakagami fading channels, Fig. 6.a shows the coverage performance of the pCA versus $K_{P}$. Our analysis closely follows the simulations. Fig. 6.a further indicates that compared to iCA, the coverage performance is slightly lower in a system with pCA. The coverage loss, however, is rather small, where $2 \leq K_{P} \leq$ 6 . The coverage performance is also shown to be slightly improved by increasing $M_{1}$.

The coverage performance vs. $\gamma$ is also plotted in Fig. 6.b. Again the analytical results closely follow the simulations and pilot-neutrality is observed for a dense HetNet.

\subsection{Designing pCA Parameters}

As indicated in Section 6.1, the coverage performance approximation given in Proposition 4 (Proposition 5) closely follows the actual system coverage performance in Rayleigh (Nakagami) fading. In the rest of this section, we consider the expressions given in these two propositions for pCA design based on Alg. 1.

For the purpose of comparison, we investigate the following systems. A benchmarking system, sys1 whose pCA parameters are obtained through an exhaustive search, sys2 whose pCA parameters are obtained by utilizing Alg. 1 . We also consider sys3, where $K_{P}=5$ 


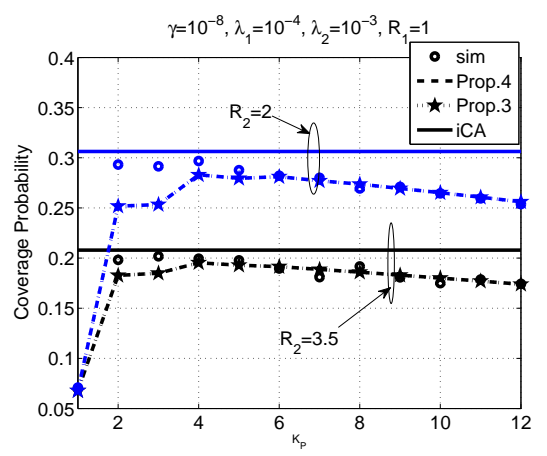

Fig. 3. Coverage probability vs. $K_{P}$

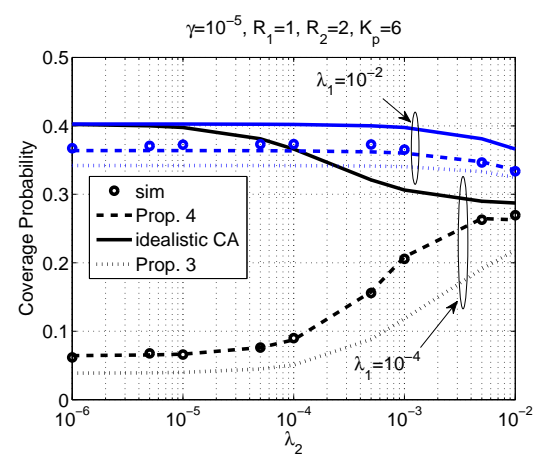

Fig. 4. Coverage probability vs. $\lambda_{2}$.

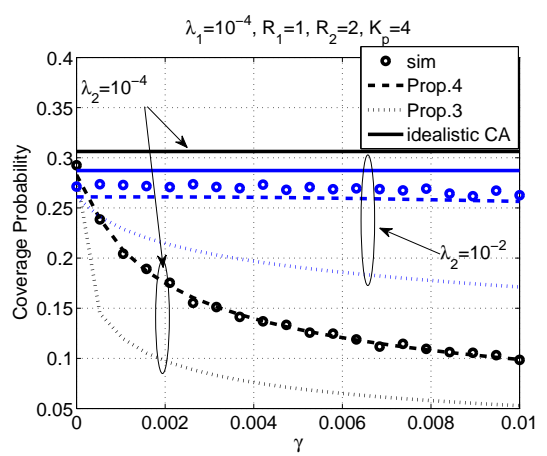

Fig. 5. Coverage probability vs. $\gamma$.

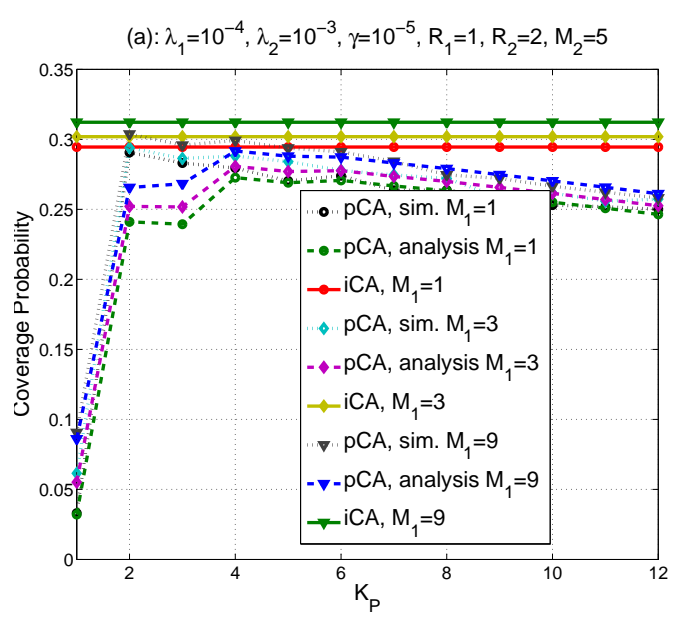

Fig. 6. Coverage performance vs. $K_{P}(\mathrm{a})$, and vs. $\gamma(\mathrm{b})$.

and the optimal partitioning is done among tiers, and finally sys4 in which for given $K_{P}=2$, each tier gets one BS in the candidate set. Note that in sys4 the closest BS across tiers is associated with the UE as the serving BS, provided that the pilot power is larger than pilot sensitivity. The CA in sys4 is in CCA with some modifications to include pilot sensitivity, pilot measurement time, and maximum SIR connectivity (in the original cCA [4,17], the typical UE simply connects to the closest BS).

Described below are our investigations for Rayleigh and Nakagami fading environments.

\subsubsection{Rayleigh Fading}

Fig. 7 plots the coverage performance of the system designed using Alg. 1 vs. $\lambda_{2}$ for several values of pilot sensitivity $\gamma$ and $\lambda_{1}$. As shown in Fig. 7, regardless of the values of $\gamma, \lambda_{1}$, and $\lambda_{2}$, sys1 achieves almost the same performance as sys2. In Table 2 we also present $K_{P}, n_{1}$, and $n_{2}$ for sys1 and sys2, showing that the same $K_{P}$, $n_{1}$, and $n_{2}$ are often obtained in both systems. In some cases, however, a larger value for $K_{P}$ is in sys2, see, e.g., $\lambda_{1}=10^{-2}$, and $\gamma=10^{-8}$. Based on the results in Table 2 , and the coverage performance observed in Fig. 7, we conclude that Alg. 1 is almost optimal in obtaining the optimal $K_{P}$ and its partitioning.

Fig. 7 plots the coverage performance of sys 3 and sys4, showing a small gap between the coverage performance

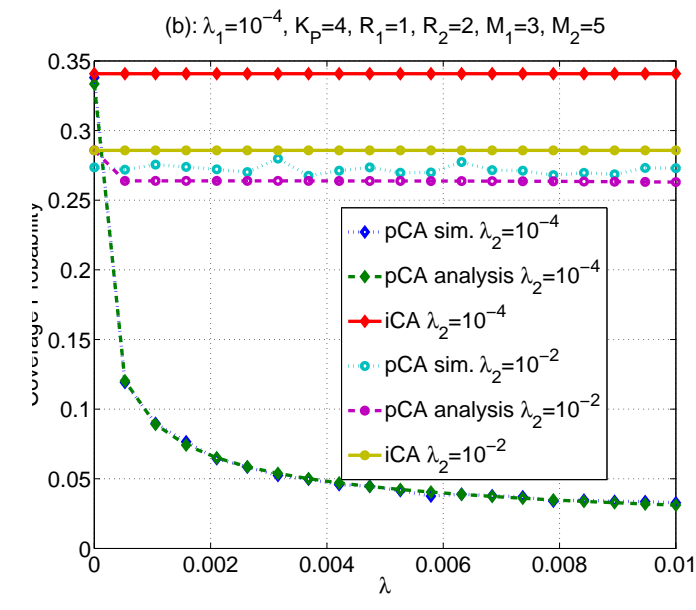

TABLE 2

$K_{P}, n_{1}$, and $n_{2}$ from exhaustive search (sys1:

$K_{P}^{\prime}=n_{1}^{\prime}+n_{2}^{\prime}$ ), and greedy algorithm (sys2:

$\left.K_{P}^{\prime \prime}=n_{1}^{\prime \prime}+n_{2}^{\prime \prime}\right)$, for $\lambda_{1}=10^{-4}$. The results are presented as: $\frac{\left[K_{P}^{\prime}, K_{P}^{\prime \prime}\right]}{\left[n_{1}^{\prime} n_{2}^{\prime}, n_{1}^{\prime \prime} n_{2}^{\prime \prime}\right]}$.

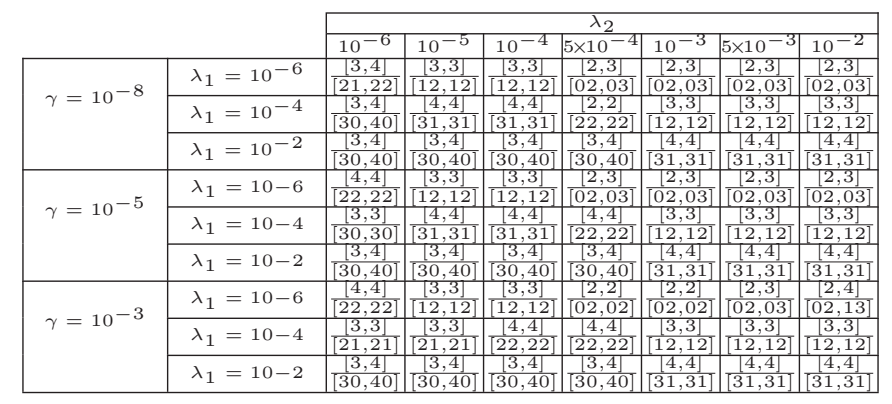

of sys3 and sys1/sys2. Fig. 7.a, however, shows a larger coverage performance degradation than sys2 for a large $\lambda_{2}$. Table 2 also shows that in both sys 2 and sys $1, K_{P} \leq 4$. Therefore, the small loss of coverage performance observed in sys3 is due mainly to fact that $K_{P}$ is larger than it should be.

The coverage probability of sys4 is also shown to be substantially smaller than that the others. A larger performance loss is seen in Fig. 7.a for a larger $\lambda_{2}$, 

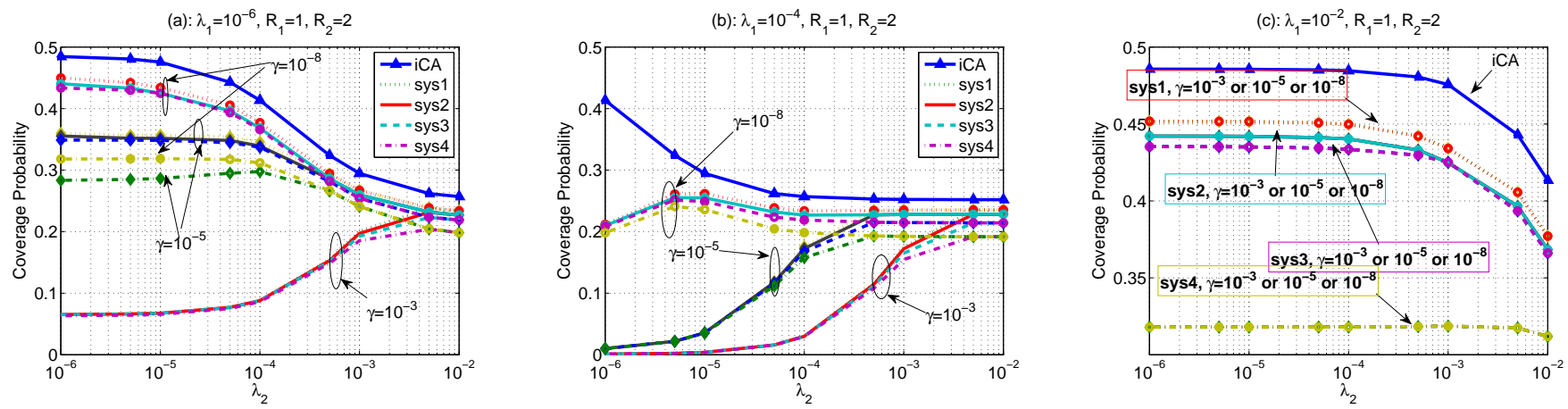

Fig. 7. Coverage probability of pCA and iCA vs. $\lambda_{2}$, for $\lambda_{1}=10^{-6}$ (a), $\lambda_{1}=10^{-4}$ (b) and $\lambda_{1}=10^{-2}$ (c).

Fig. 7.b, where $\gamma=10^{-8}$ and $\gamma=10^{-5}$, and Fig. 7.c for all values of $\gamma$. Fig. 7.c further confirms pilot-neutrality for dense HetNets.

\subsubsection{Nakagami Fading}

For Nakagami fading, we investigate the coverage performance of sys2. Fig. 8 plots the coverage probability against $M_{1}$, and $M_{2}$. For $\lambda_{1}=10^{-6}$, the coverage probability reduces by increasing $M_{2}$ and is almost independent of $M_{1}$. Note that $\lambda_{2}>\lambda_{1}$, and therefore, the ICI component is also increased by increasing $M_{2}$. For the case of $\lambda_{1}=10^{-2}$, which is larger than $\lambda_{2}$, the coverage probability demonstrates stability, where $M_{2}$ is changed, while it is reduced by increasing $M_{1}$.

On the other hand, for given $\gamma=10^{-3}$ and for small values of $M_{1}$, the coverage probability is increased by increasing $\lambda_{1}$ irrespective of $M_{2}$. However, increasing $M_{1}$, reduces the level of growth in the coverage performance.

We further present values of $n_{1}$ and $n_{2}$ versus $M_{1}$ and $M_{2}$ in Figs. 9, and 10, respectively. For $\lambda_{1}=10^{-6}<\lambda_{2}$, irrespective of the values of $M_{1}, M_{2}$ and $\gamma$, none of the BSs in tier 1 is chosen in CA. Fig. 10 also shows that for $\gamma=10^{-8}, M_{2}<2$, and any value of $M_{1}$, we obtain $n_{2}=$ 3 , which reduces to $n_{2}=2$ by further increasing $M_{2}$. These suggest that in systems under Nakagami fading, we might require a smaller $K_{P}$ than to Rayleigh fading.

For $\lambda_{1}=10^{-2}>\lambda_{2}$, one can see that in general, $n_{2}=0$ except in the case of $M_{1}=1$ (and any value of $M_{2}$ ) which requires $n_{2}=1$. Further, Fig. 9 shows that for a large $\lambda_{1}$, the larger the value of $M_{1}$, the smaller $n_{1}$. In fact, by increasing $M_{1}$, the impact of fading fluctuations is reduced in general, so it is sufficient to consider $K_{P}=K$ BSs only for CA. In this example, since $\lambda_{1} \gg \lambda_{2}$, all BSs for $\mathrm{CA}$ are chosen from tier 1.

\section{Conclusions}

This paper addressed the incorporation of practical cell association (pCA) in the modeling and analysis of large-scale heterogeneous cellular networks (HetNets). We mathematically modeled pCA by considering actual traits and limitations of pilot measurements in the maxSIR CA rule. Specifically, we considered pilot powers, portion of time slot designated for pilot measurement, pilot sensitivity at the UEs, and the number of prescribed measurable pilots $K_{P}$. The proposed model is could be tailored to the idealistic CA model in [7] or to a modified version of the closest CA model of [17].

Adopting tools from stochastic geometry and PPP, we then evaluated the coverage probability under the pCA model as a function of CA's and network's parameters. Various approximations are obtained for both cases of Rayleigh and Nakagami fading and their accuracies are corroborated with simulations. We further exploited our analysis to obtain the optimal $K_{P}$ and its corresponding partitioning among tiers in order to maximize the coverage performance of the HetNet. A greedy algorithm was developed and its efficiency was also confirmed against exhaustive search. For both Rayleigh and Nakagami fading environments there exist an optimal size of the candidate set, $K_{P}$, that minimizes the coverage loss of the HetNet with pCA compared to the iCA. Furthermore, we showed that in Nakagami fading environments, a smaller number of pilot measurements is required to achieve the optimized coverage probability with Nakagami fading than Rayleigh fading.

Finally, we uncovered various interesting features of dense HetNets: dense HetNets (i) are pilot-neutral, and (ii) scale invariancy in HetNets with iCA is not sustained under the pCA model.

\section{REFERENCES}

[1] J. G. Andrews et al., "What will 5G be?" IEEE JSAC, vol. 32, no. 6, pp. 1065-1082, Jun. 2014

[2] F. Boccardi et al., "Five disruptive technology directions for 5G," IEEE Comm. Mag., vol. 54, no. 2, pp. 74-80, Feb. 2014.

[3] K. Shen and W. Yu, "Distributed pricing-based user association for downlink heterogeneous cellular networks," IEEE JSAC, vol. 32, no. 6, pp. 1100-1113, Jun. 2014.

[4] H. S. Jo et al., "Heterogeneous cellular networks with flexible cell association: A compehensive downlink SINR analysis," IEEE Trans. Wireless Comm., vol. 11, no. 10, pp. 3484-3495, Oct. 2012.

[5] K. Son et al., "Dynamic association for load balancing and interference avoidance in multi-cell networks kyuho son, student member," IEEE Trans. Wireless Comm., vol. 8, no. 7, pp. 3566-3576, Jul. 2009.

[6] R. Sun, "Joint downlink base station association and power control for max-min fairness: Computation and complexity," IEEE JSAC, vol. 33, no. 6, pp. 1040 - 1054, Jun. 2015. 


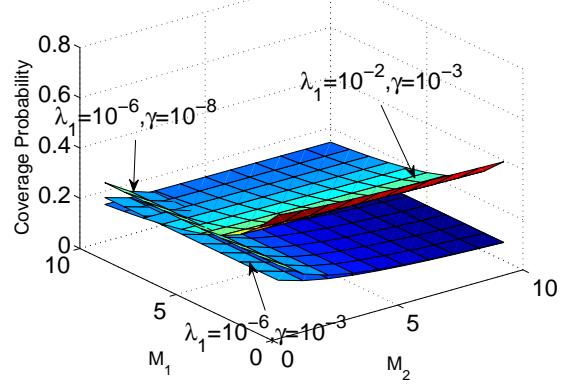

Fig. 8. Coverage Probability vs. $M_{1}$ Fig. 9 and $M_{2}$ for $\lambda_{2}=10^{-3}$.
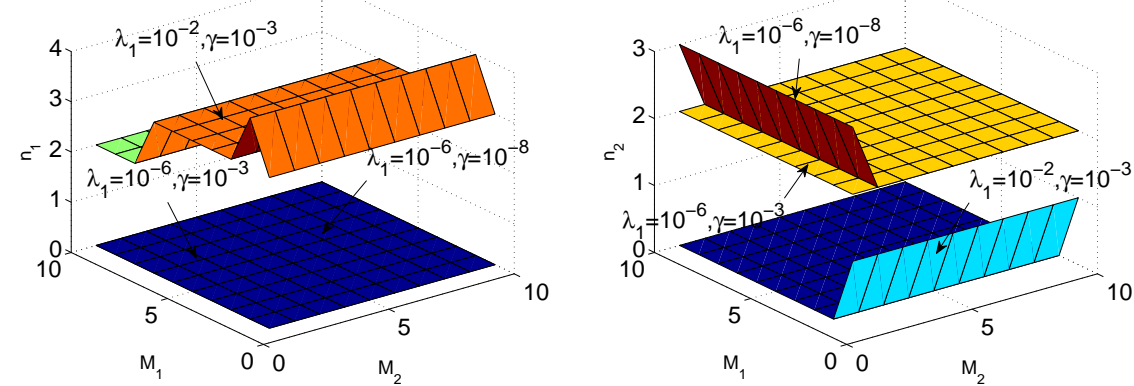

Fig. 10. $n_{2}$ vs. $M_{1}$ and $M_{2}$ for $\lambda_{2}=$ $10^{-3}$.
[7] H. S. Dhillon et al., "Modeling and analysis of $k$-tier downlink heterogeneous cellular network," IEEE JSAC, vol. 30, no. 3, pp. 550-560, Apr. 2012.

[8] _ - "Downlink MIMO hetnets: Modeling, ordering results and performance analysis," IEEE Trans. Wireless. Comm., vol. 12, no. 10, pp. 5208-5222, Oct. 2012.

[9] R. Cai et al., "Cost-efficient optimization of base station densities for multitier heterogeneous cellular networks," IEEE Trans. Wireless. Comm., vol. 15, no. 3, pp. 2381-2393, Mrc. 2016.

[10] j. Wen et al., "On the capacity of downlink multi-hop heterogeneous cellular networks," IEEE Trans. Wireless. Comm., vol. 13, no. 8, pp. 4092-4103, Aug. 2014.

[11] M. Haenggi et al., "Stochastic geometry and random graphs for the analysis and design of wireless networks," IEEE JSAC, vol. 27, no. 7, pp. 1029-1046, Sep. 2009.

[12] H. ElSawy et al., "Modeling and analysis of cellular networks using stochastic geometry: A tutorial," 2016, [Online]. Available: http://arxiv.org/abs/1604.03689.

[13] W. Lu and M. D. Renzo, "Stochastic geometry modeling of cellular networks: Analysis, simulation and experimental validation," 2015, [Online]. http://arxiv.org/abs/ 1506.03857.

[14] A. Guo and M. Haenggi, "Spatial stochastic models and metrics for the structure of base stations in cellular networks," IEEE Trans. Wireless Comm., vol. 12, no. 11, pp. 5800-5812, Nov. 2013.

[15] H. Wei et al., "Approximate SIR analysis in general heterogeneous cellular networks," IEEE Trans. Comm., vol. 64, no. 3, pp. 12591273, Mrc. 2016

[16] R. K. Ganti and M. Haenggi, "Asymptotics and approximation of the SIR distribution in general cellular networks," IEEE Trans. Wireless. Comm., vol. 15, pp. 2130-2143, Mrc. 2016.

[17] J. G. Andrews et al., "A tractable approach to coverage and rate in cellular networks," IEEE Trans. on Comm., vol. 59, no. 11, pp. 3122-3134, Nov. 2011

[18] A. Ghosh et al., "Heterogeneous cellular networks: from theory to practice," IEEE Comm. Mag., vol. 50, no. 6, pp. 54-64, Jun. 2012.

[19] B. Blaszczyszyn et al., "Wireless networks appear Poissonian due to strong shadowing," IEEE Trans. Wireless Comm., vol. 14, no. 8 , pp. 4379-4390, Aug. 2015.

[20] P. Madhusudhanan et al., "Analysis of downlink connectivity models in aheterogenous cellular network via stochastic geometry," IEEE Trans. Wireless. Comm., vol. 15, no. 6, pp. 3895-3907, Jun. 2016.

[21] H. P. Keeler et al., "SINR-based k-coverage probability in cellular networks with arbitrary shadowing," in Proc. IEEE international symposium on information theory, 2013, Available at http://eprints.lancs.ac.uk/79820/1/K-tier-MIMO-MRCVTC.pdf.

[22] B. Blaszczyszyn and H. P. Keeler, "Studying the SINR process of the typical user in poisson networks using its factorial moment measures," IEEE Trans. Information Theory, vol. 61, no. 12, pp. 6774-6794, Dec. 2015.

[23] P. Herath et al., "Coverage and rate analysis for limited information cell association in stochastic-layout cellular networks," to appear IEEE Trans. Veh. Technol., 2016.

[24] A. Sankaraman et al., "Performance-oriented association in large cellular networks with technology diversity," CoRR, vol. arXiv:1603.06928, 2016, [Online] http://arxiv.org/abs/1603.06928.

[25] S. Mukherjee, "Distribution on downlink SINR in heterogeneous cellular network," IEEE JSAC, vol. 30, no. 3, pp. 575-585, Apr 2012.

[26] Y. Lin et al., "Optimizing user association and spectrum allocation in hetnets: A utility perspective," IEEE JSAC, vol. 33, no. 6, pp. 1025-1039, Jun. 2015

[27] R. Madan et al., "Cell association and interference coordination in heterogeneous LTE-A cellular networks," IEEE JSAC, vol. 28 no. 12, pp. 1479-1489, Dec. 2010.

[28] L. P. Qian et al., "Joint base station association and power control via benders decompostition," IEEE Trans. Wireless Comm., vol. 4 , no. 12, pp. 1651-1665, Apr. 2013.

[29] y. Wang et al., "Load-aware dynamic biasing cell association in small cell networks," IEEE ICC'14-Mobile and Wireless Networking Symposium, pp. 2684-2689, 2014

[30] C. Li et al., "Analysis of area spectral efficiency and link reliability in multiuser MIMO HetNets," in Proc. IEEE Int. Conf. Commun. (ICC), Jun. 2015

[31] V. Chandrasekhar et al., "Coverage in multi-antenna two-tier networks," IEEE Trans. Wireless. Comm., vol. 8, no. 10, pp. 53145327, Oct. 2009

[32] M. G. Khoshkholgh et al., "Performance evaluation of MISO-SDMA in heterogenous networks with practical cell association," in Proc. IEEE VTC-Fall, 2016, Available at http://eprints.lancs.ac.uk/79817/1/K-tier-MIMO-SDMA-HCNVTC.pdf.

[33] R. H. Y. Louie et al., "Open-loop spatial multiplexing and diversity communications in ad hoc networks," IEEE Trans. Inf. Theory, vol. 57, no. 1, pp. 317-344, Jan. 2011.

[34] Y. Wu et al., "Generalized framework for the analysis of linear MIMO transmission schemes in decentralized wireless Ad Hoc networks," IEEE Trans. Wireless. Comm., vol. 11, no. 8, pp. 28152827, Aug. 2012.

[35] R. Vaze and R. W. H. Jr., "Transmission capacity of ad-hoc networks with multiple antennas using transmit stream adaptation and interference cancellation," IEEE Trans. Inf. Theory, vol. 58, no. 2, pp. 780-792, Feb. 2012.

[36] M. G. Khoshkholgh et al., "Coverage performance of MIMOMRC in heterogeneous networks: a stochastic geometry perspective," in Proc. IEEE VTC-Fall, 2016, Available at http:/ /eprints.lancs.ac.uk/79820/1/K-tier-MIMO-MRCVTC.pdf.

[37] M. G. Khoshkholgh and V. C. M. Leung, "On the performance of MIMO-SVD multiplexing systems in HetNets: A stochastic geometry perspective," to appear in IEEE Trans. TVT, 2017.

[38] A. Shojaeifard et al., "Exact SINR statistics in the presence of heterogeneous interferers," IEEE Trans. Inf. Theory, vol. 61, no. 12, pp. 6759-6773, Dec. 2015.

[39] U. Schilcher et al., "Interference functionals in poisson networks," IEEE Trans. Inf. Theory, vol. 62, no. 1, pp. 370-383, Jan. 2016.

[40] X. Zhang and J. G. Andrews, "Downlink cellular network analysis with multi-slope path loss models," IEEE Trans. Comm., vol. 63, no. 5, pp. 1881-1894, May 2015.

[41] M. D. Renzo et al., "The intensity matching approach: A tractable stochastic geometry approximation to system-level analysis of 
cellular networks," IEEE Trans. Wireless Comm., vol. 15, no. 9, pp. 5963-5983, Sep. 2016.

[42] M. Ding and D. Lopez-Perez, "On the performance of practical ultra-dense networks: The major and minor factors," CoRR, vol. arXiv:1701.07964, 2017, [Online]. https://arxiv.org/abs/1701.07964?context=math.

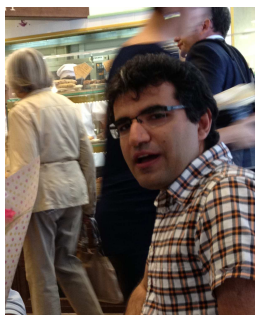

Mohammad G. Khoshkholgh received his B.Sc. degree in Electrical Engineering from Isfahan University, Isfahan, Iran, in 2006, his M.Sc. degree in Electrical Engineering from the Tarbiat Modares University, Tehran, Iran, in 2008. $\mathrm{He}$ was with Wireless Innovation Laboratory in Tarbiat Modares University from 2008 until 2012. From February 2012 to February 2014 he was with Simula Research Laboratory, Fornebu, Norway working on developing communication solutions for smart grid systems. He is now with the University of British Columbia. His research interests are mainly in wireless communications, radio resource allocations, and spectrum sharing.

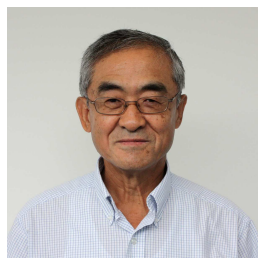

Kang G. Shin is the Kevin \& Nancy O'Connor Professor of Computer Science in the Department of Electrical Engineering and Computer Science, The University of Michigan, Ann Arbor. His current research focuses on QoS-sensitive computing and networking as well as on embedded real-time and cyber-physical systems.

He has supervised the completion of $79 \mathrm{PhDs}$, and authored/coauthored more than 900 technical articles (more than 300 of these are in archival journals), a textbook and more than 40 patents or invention disclosures, and received numerous best paper awards, including the Best Paper Awards from the 2011 ACM International Conference on Mobile Computing and Networking (MobiCom'11), the 2011 IEEE International Conference on Autonomic Computing, the 2010 and 2000 USENIX Annual Technical Conferences, as well as the 2003 IEEE Communications Society William R. Bennett Prize Paper Award and the 1987 Outstanding IEEE Transactions of Automatic Control Paper Award. He has also received several institutional awards, including the Research Excellence Award in 1989, Outstanding Achievement Award in 1999, Distinguished Faculty Achievement Award in 2001, and Stephen Attwood Award in 2004 from The University of Michigan (the highest honor bestowed to Michigan Engineering faculty); a Distinguished Alumni Award of the College of Engineering, Seoul National University in 2002; 2003 IEEE RTC Technical Achievement Award; and 2006 Ho-Am Prize in Engineering (the highest honor bestowed to Korean-origin engineers).

He was a co-founder of a couple of startups and also licensed some of his technologies to industry.

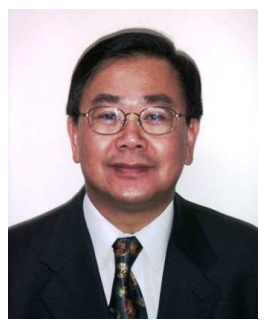

Victor C. M. Leung [S'75, M'89, SM'97, F'03] received the B.A.Sc. (Hons.) degree in electrical engineering from the University of British Columbia (UBC) in 1977, and was awarded the APEBC Gold Medal as the head of the graduating class in the Faculty of Applied Science. $\mathrm{He}$ attended graduate school at UBC on a Natural Sciences and Engineering Research Council Postgraduate Scholarship and completed the Ph.D. degree in electrical engineering in 1981.

From 1981 to 1987 , Dr. Leung was a Senior Member of Technical Staff and satellite system specialist at MPR Teltech Ltd., Canada. In 1988, he was a Lecturer in the Department of Electronics at the Chinese University of Hong Kong. He returned to UBC as a faculty member in 1989, and currently holds the positions of Professor and TELUS Mobility Research Chair in Advanced Telecommunications Engineering in the Department of Electrical and Computer Engineering. Dr. Leung has co-authored more than 700 technical papers in international journals and conference proceedings, 29 book chapters, and co-edited 8 book titles. Several of his papers had been selected for best paper awards. His research interests are in the areas wireless networks and mobile systems.

Dr. Leung is a registered professional engineer in the Province of British Columbia, Canada. He is a Fellow of IEEE, the Royal Society of Canada, the Engineering Institute of Canada, and the Canadian Academy of Engineering. He was a Distinguished Lecturer of the IEEE Communications Society. He is a member of the editorial boards of the IEEE Wireless Communications Letters, Computer Communications, and several other journals, and has previously served on the editorial boards of the IEEE Journal on Selected Areas in Communications Wireless Communications Series, IEEE Transactions on Wireless Communications, IEEE Transactions on Vehicular Technology, IEEE Transactions on Computers, and Journal of Communications and Networks. He has guest-edited many journal special issues, and contributed to the organizing committees and technical program committees of numerous conferences and workshops. He was a recipient of the IEEE Vancouver Section Centennial Award and 2012 UBC Killam Research Prize. 